\title{
Positive solutions to the singular $p$-Laplacian BVPs with sign-changing nonlinearities and higher-order derivatives in Banach spaces on time scales
}

\author{
Youhui Su and Zhaosheng Feng
}

Communicated by Y. Charles Li, received Feburary 1, 2011.

\begin{abstract}
In this paper, in order to establish the existence criteria for positive solutions of a multiple-point Dirichlet-Robin BVPs in Banach spaces on time scales, first we prove a fixed point theorem on monotone operators and the Ascoli-Arzela's theorem on time scales. Then using the monotone operator method, we explore the existence criteria of positive solutions for a general multiple-point Dirichlet-Robin BVPs in Banach spaces on time scales with the singular sign-changing nonlinearities and higher-order derivatives. Finally an example is illustrated to indicate the application of our main results, which generalize some well-known results in the literature.
\end{abstract}

\section{Contents}

1. Introduction 150

2. Preliminaries 152

3. Some Definitions and Theorems on Time Scales 154

4. Existence of Positive Solutions 157

5. Construction of $\alpha(t)$ and $\beta(t) \quad 164$

6. One Example 169

$\begin{array}{ll}\text { References } & 170\end{array}$

1991 Mathematics Subject Classification. Primary 34B15, 35B09; Secondary 34A12.

Key words and phrases. boundary value problem, time scales, fixed point, delta derivative, positive solution, partial ordering, $p$-Laplacian, Ascoli-Arzela's Theorem.

The work is supported by NSFC (10771212), the Department of Education Jiangsu Province Grant (09KJD110006) and the Fundamental Research Funds for the Central Universities Grant (2010QNA40). 


\section{Introduction}

Since the work by Hilger [1] in 1988, it seems that the time scales calculus has attracted much attention. The time scales approach not only unifies differential and difference equations, but also solves some other problems powerfully, such as a mix of stop-start and continuous behaviors $[\mathbf{2}, \mathbf{3}]$. Nowadays the theory on time scales has been widely applied to several scientific fields such as biology, heat transfer, stock market, wound healing and epidemic models [2-5] etc. Recently, considerable works have been undertaken in the existence problems of solutions to dynamic systems on time scales, for details, see $[\mathbf{6}-\mathbf{1 3}]$ and the references therein.

Consider the $p$-Laplacian operator $\varphi_{p}(u)$ :

$$
\varphi_{p}(u)= \begin{cases}u^{p-1}, & u \geq \theta, \\ (-1)^{p-2} u^{p-1}, & u<\theta,\end{cases}
$$

and

$$
\left(\varphi_{p}\right)^{-1}=\varphi_{q}
$$

where $p>1$ and $1 / p+1 / q=1$. We make the blanket assumption that 0 and $T$ are points in $\mathbb{T}$ and the interval $(0, T)_{\mathbb{T}}$ represents $(0, T) \cap \mathbb{T}$. Other types of interval can be defined in a similar way. Let us briefly recall some relevant results about the existence of solutions to the $p$-Laplacian boundary value problems (BVPs). For the existence of positive solutions to the BVPs with sign changing nonlinearities on differential equations, there is a number of works, for example, [14-16] etc concerning this problem by using the method of upper and lower solutions and the critical point theory. For the existence of positive solutions to the singular $p$ Laplacian $m$-point BVPs with sign changing nonlinearities on time scales, sufficient conditions are established through developing various fixed point theory and the iterative approach $[\mathbf{9}, \mathbf{1 0}]$. Some results in $[\mathbf{9}, \mathbf{1 0}, \mathbf{1 4}-\mathbf{1 6}]$ may be of interest to those who study positive solutions of boundary value problems as well as to those who study non-Newtonian fluid theory and turbulent flows of gases in porous media.

In order to better understand the background of the singular $p$-Laplacian $m$ point BVPs, here we would like to review some results in our previous works $[\mathbf{9}, \mathbf{1 0}]$. In $[\mathbf{9}], \mathrm{Su}$ et.al investigated the following $m$-point singular $p$-Laplacian BVPs on time scales

$$
\begin{gathered}
\left(\varphi_{p}\left(u^{\Delta}(t)\right)\right)^{\nabla}+q(t) f(t, u(t))=0, t \in(0, T)_{\mathbb{T}}, \\
u(0)=0, u(T)-\sum_{i=1}^{m-2} \psi_{i}\left(u\left(\xi_{i}\right)\right)=0,
\end{gathered}
$$

where $f(t, x):[0, T]_{\mathbb{T}} \times(0, \infty) \rightarrow \mathbb{R}$ is continuous and the singularity may occur at $u=0$. Some existence criteria for positive solutions of the BVPs (1.1) are established by using the Schauder fixed point theorem and by extending the method of upper and lower solutions. In [10], Su et.al discussed the following $m$-point singular $p$-Laplacian the BVPs with the sign changing nonlinear terms on a time scale $\mathbb{T}$ :

$$
\begin{aligned}
& \left(\varphi_{p}\left(u^{\Delta}(t)\right)\right)^{\nabla}+q(t) f\left(t, u(t), u^{\Delta}(t)\right)=0, t \in(0, T)_{\mathbb{T}}, \\
& u(0)=0, \sum_{j=1}^{m_{1}} \phi_{j}\left(u\left(\xi_{j}^{*}\right)\right)-\sum_{i=1}^{m_{2}} \psi_{i}\left(u^{\Delta}\left(\xi_{i}\right)\right)=0, m_{1}, m_{2} \in\{1,2, \ldots\},
\end{aligned}
$$

where $f(t, x, y):(0, T)_{\mathbb{T}} \times(0, \infty) \times \mathbb{R} \rightarrow \mathbb{R}$ is continuous, and the singularity may occur at $u=0, t=0$ and $t=T$. Some existence criteria for positive solutions of the general multi-point Dirichlet-Robin BVPs (1.2) are presented by using the monotone iterative method. 
It is notable that results on time scales in $[\mathbf{6}-\mathbf{1 3}]$ are mainly concerned with the existence of positive solutions to the BVPs with the nonlinear terms which do not involve the higher-order derivatives explicitly and the nonlinear function $f$ has continuity (rd-continuity), so that the corresponding solution operator has continuity (rd-continuity). It is obvious that weakening the existence conditions of the BVPs has been an interesting subject in differential equations and related dynamical systems, especially when the function $f(t, x, y)$ or $f(t, x)$ is only continuous with respect to $t$ in Banach spaces. So far, to the best of our knowledge, it appears that there is few literature which considers the existence of positive solutions to the singular $p$-Laplacian BVPs with sign-changing nonlinearities involving the higher-order derivatives on time scales in Banach spaces, although there are a vast number of works [17-19] etc to study the existence of solutions to BVPs on differential equations in Banach spaces. Therefore, it is natural and necessary to do extensive research on the singular $p$-Laplacian BVPs on time scales in Banach spaces. Here we focus on the existence of positive solutions for the $p$-Laplacian dynamic equations with the nonlinear terms involving the higher-order derivatives explicitly in Banach spaces on time scales and the nonlinearity is only continuous with respect to $t$.

More precisely, in this paper we consider the multiple-point singular $p$-Laplacian BVPs in the compact Banach space $E_{1}$ on time scales:

$$
\begin{gathered}
\left(\varphi_{p}\left(u^{\Delta}(t)\right)\right)^{\nabla}+q(t) f\left(t, u(t), u^{\Delta}(t), \ldots, u^{\Delta^{n}}(t)\right)=\theta, \quad t \in(0, T)_{\mathbb{T}}, \\
u(0)=\theta, \quad \sum_{j=1}^{m_{1}} \phi_{j}\left(u\left(\xi_{j}^{*}\right)\right)-\sum_{i=1}^{m_{2}} \psi_{i}\left(u^{\Delta}\left(\xi_{i}\right)\right)=0, \quad m_{1}, m_{2} \in\{1,2, \ldots\},
\end{gathered}
$$

where $\theta$ is a zero element of $E_{1}, \phi_{j}$ and $\psi_{i}: E_{1} \rightarrow \mathbb{R}$ are nondecreasing, $\phi_{j}$ and $\psi_{i}$ may be nonlinear, and $0<\xi_{1}^{*}<\xi_{2}^{*}<\ldots<\xi_{m_{1}-1}^{*}<\xi_{m_{1}}^{*}=T$ and $0<\xi_{1}<\xi_{2}<\ldots<\xi_{m_{2}} \leq T$. The function $f:(0, T)_{\mathbb{T}} \times P_{1} \times E_{1}^{n} \rightarrow E_{1}$ is continuous with respect to $t$, where $P_{1}=\{u \in X \mid u \geq \theta\}$ and $X=C\left([0, T]_{\mathbb{T}}, E_{1}\right) \cap$ $C^{\Delta}\left((0, T]_{\mathbb{T}^{\kappa}}, E_{1}\right) \cap \ldots \cap C^{\Delta^{n}}\left((0, T]_{\mathbb{T}^{\kappa}}, E_{1}\right)$. The singularity may occur at $u=\theta$, $t=0$ or $t=T$, and the nonlinearity is allowed to change sign and contain the higherorder derivatives explicitly. It is continuous with respect to $t$ only. Apparently, the boundary condition (1.4) includes the Dirichlet boundary condition and the Robin boundary condition as particular cases. By applying a monotone operator method, we obtain some new existence criteria for positive solutions of the BVPs (1.3)-(1.4) in Banach spaces on time scales. Our results are even new for the corresponding differential equations $(\mathbb{T}=\mathbb{R})$ and difference equations $(\mathbb{T}=\mathbb{Z})$, as well for the general time scales setting.

As an application, an example is given to illustrate our main results. In particular, if $f\left(t, u, u^{\Delta}, \ldots, u^{\Delta^{n}}\right)=f(t, u)$, our results generalize some known results in [14] when $\mathbb{T}=\mathbb{R}$ and $E_{1}=\mathbb{R}$, and extend some results in [16] when $p=2$. When $f\left(t, u, u^{\Delta}, \ldots, u^{\Delta^{n}}\right)=f(t, u)$ and $E_{1}=\mathbb{R}$, our results improve some existing results in $[\mathbf{7}, \mathbf{9}]$. When $f\left(t, u, u^{\Delta}, \ldots, u^{\Delta^{n}}\right)=f\left(t, u, u^{\Delta}\right)$ and $E_{1}=\mathbb{R}$, our results also generalize the recent results in $[\mathbf{1 0}]$.

This paper is organized as follows. In Section 2, we introduce some useful definitions and prove a fixed point theorem on monotone operators in Banach spaces. In Section 3, we present some definitions and theorems in Banach spaces on time scales. In particular, the Ascoli-Arzela's theorem is generalized to Banach spaces on time scales. In Section 4, by using the monotone operator method, we obtain 
the existence of positive solutions of the problem (1.3) and (1.4). In Section 5, we introduce a technical method to show how to construct a lower solution and an upper solution under certain conditions. In Section 6, as an application, we present an examples to illustrate our main results.

Throughout this paper, we assume that

(H1): $f\left(t, x_{0}, x_{1}, \ldots, x_{n}\right):(0, T)_{\mathbb{T}} \times P_{1} \times E_{1}^{n} \rightarrow E_{1}$ is continuous with respect to $t$, where $E_{1}^{n}=E_{1} \times E_{1} \ldots E_{1}$ and $E_{1}$ is a compact Banach space;

(H2): $q(t):(0, T)_{\mathbb{T}} \rightarrow(0, \infty)$ is continuous and satisfies $\int_{0}^{T} q(t) \nabla t<\infty$;

(H3): $\phi_{j}$ and $\psi_{i}: E_{1} \rightarrow \mathbb{R}$ are continuous and nondecreasing, where $j=$ $1,2, \ldots, m_{1}$ and $i=1,2, \ldots, m_{2}$.

We need these three assumptions to prove the existence of positive solutions of system (1.3)-(1.4) when we use the lower and upper solutions for the nonsingular problem.

\section{Preliminaries}

In order to present our results in a straightforward manner, we start with several basic definitions and useful theorems, which can be found in references $[\mathbf{2 0 - 2 3}]$. Then we prove the existence criteria for monotone operators.

Let $E$ be a real Banach space and the norm in $E$ is denoted as $\|\cdot\|_{E}$.

Definition 2.1. [20, 21] Let $E$ be a real Banach space and $\leq$ is a partial ordering defined in E. Suppose that

(i) if $a \leq b \in E$, then the set $[a, b]=\{v \in E \mid a \leq v \leq b\}$ is an order interval in $E$;

(ii) $A$ is a monotone operator in $E$ if and only if

$$
v, w \in E \text { and } v \leq w .
$$

Then $A v \leq A w$ holds.

Let $E$ be a partial ordering set with $\leq$ and $A_{1} \subset E$. If for $\forall x, y \in A_{1}$, we have $x \leq y$ or $x \geq y$, then we say that $A_{1}$ is a complete partial ordering set. When $x_{0} \in E$, we say that $x_{0}$ is a maximum element in $A_{1}$ if $x \leq x_{0}$ for $\forall x \in A_{1}$.

Theorem 2.2. $\quad[\mathbf{2 0}, \mathbf{2 2}]$ (Zorn's Lemma) Let $\mathrm{X}$ be a partial ordering set. If every complete partial ordering set of $X$ is super bounded in $X$, then $X$ has a maximum element.

Definition 2.3. $[\mathbf{2 0}, \mathbf{2 2}]$ Let $E$ be a real Banach space. A nonempty, closed, and convex set $P \subset E$ is said to be a cone provided that the following two conditions are satisfied:

(i) if $x \in P$ and $\lambda \geq 0$, then $\lambda x \in P$;

(ii) if $x \in P$ and $-x \in P$, then $x=0$.

Let $A: E \rightarrow E$ be an operator. We have two definitions here:

Definition 2.4. $[\mathbf{2 0 , 2 1}]$ We say that $u$ is a fixed point of $A$ if $u=A u$; $u^{*}$ is said to be a minimal fixed point of $A$, if there exist a fixed point $u_{0}$ of $A$ such that $u_{0} \leq u^{*}$, then we have $u_{0}=u^{*}$. Similarly, we can define the maximal fixed point of $A$.

Definition 2.5. $[\mathbf{2 0}, \mathbf{2 1}]$ An ordered Hausdorff topological space $X_{1}$ with ordering $\leq$, is an ordering compatible space. If both $\left\{a_{n}\right\}$ and $\left\{b_{n}\right\}\left(a_{n} \leq b_{n}\right)$ are convergent sequences in $X_{1}$ with limits $a$ and $b$ respectively, then we have $a \leq b$. 
If $E$ possesses a cone, then $E$ is an ordering compatible space. Now let us prove the following theorem which plays a crucial role in the proof of our main results described in the next section.

TheOREM 2.6. Let $P \subset E$ be a cone and $E$ be a Banach space with the partial ordering induced by $P$. Assume that $u_{0}, v_{0} \in E, u_{0} \leq v_{0}, D=\left[u_{0}, v_{0}\right]$, and $A$ : $D \rightarrow E$ satisfies the following conditions:

(i) $A$ is a monotone operator;

(ii) $A(D)$ is compact in $E$.

If $u_{0} \leq A u_{0}$ and $A v_{0} \leq v_{0}$, then there exist a minimal fixed point $x_{*}$ and a maximal point $x^{*}$ of $A$ in $\left[u_{0}, v_{0}\right]$ respectively. Moreover, if $u_{n}=A u_{n-1}$ and $v_{n}=A v_{n-1}$, $n=1,2, \ldots$, then we have

$$
u_{0} \leq u_{1} \leq \ldots \leq u_{n} \leq \ldots \leq v_{n} \leq \ldots \leq v_{1} \leq v_{0},
$$

and

$$
x_{*}=\lim _{n \rightarrow \infty} u_{n}, \quad x^{*}=\lim _{n \rightarrow \infty} v_{n}
$$

Proof of Theorem 2.6. Set $G=\{x \in D \mid x \leq A x\}$. Note that $G$ is nonempty and $u_{0} \in G$. Letting $M$ be a complete partial ordering set in $G$, we will prove that $M$ has a bound in $G$. According to conditions of Theorem 2.6, $A(D)$ is precompact and $A(D)$ is separable. Suppose that $\left\{y_{n}\right\}$ is dense in $A(D)$. Let $y_{1}=z_{1}$ and $z_{n}=\max \left\{z_{n-1}, y_{n}\right\}, n=2,3, \ldots$. Since $M$ is a complete partial ordering set, we have $\left\{z_{n}\right\} \subset A(M)$ and

$$
z_{1} \leq z_{2} \leq \ldots \leq z_{n} \leq \ldots
$$

By condition $(i i)$, we see that $\left\{z_{n}\right\}$ is compact, so there exists a $\bar{z} \in E$ such that $z_{n} \rightarrow \bar{z}$ as $n \rightarrow \infty$. For $\forall n \in \mathbb{N}$ we have

$$
z_{n} \leq \bar{z} \text { and } y_{n} \leq z_{n} \leq \bar{z} .
$$

For $\forall y \in A(M)$, there exists $\left\{y_{n_{k}}\right\} \subset\left\{y_{n}\right\}$ such that $y_{n_{k}} \rightarrow y$ as $k \rightarrow \infty$. According to $(2.1)$, we find

$$
y \leq \bar{z}, \forall y \in A(M) .
$$

Since $M \subset D=\left[u_{0}, v_{0}\right]$, we have $\left\{z_{n}\right\} \subset A(M) \subset A(D) \subset\left[A u_{0}, A v_{0}\right]$, and $A u_{0} \leq$ $z_{n_{k}} \leq A v_{0}$. This implies $\bar{z} \in D$. For $\forall x \in M$ and $A x \in A(M)$, according to $(2.2)$ we have $x \leq A x \leq \bar{z}$, so $\bar{z}$ is a supper bound of $M$.

Next we prove that $\bar{z} \in G$. We know that $u_{0} \leq \bar{z} \leq v_{0}$. Let $z_{n}=A x_{n}$ when $x_{n} \in M$. Then we have $x_{n} \leq A x_{n}=z_{n} \leq \bar{z}$. Since $A$ is increasing, we have $z_{n}=A x_{n} \leq A \bar{z}$. By Definition 2.5, we deduce $\bar{z} \leq A \bar{z}$ and $\bar{z} \in G$. According to Zorn's Lemma, there exists a $x^{*} \in G$ such that for $\forall x \in G$ we have $x \leq x^{*}$. Since $x^{*} \in G, x^{*} \leq A x^{*}$ and $A x^{*} \leq A\left(A x^{*}\right)$ hold, it implies that $A x^{*} \in G$ and $x^{*}<A x^{*}$. This yields a contradiction. Thus, we have $x^{*}=A x^{*}$.

Finally, we prove that there exist a maximal fixed point and a minimal fixed point of $A$ in $E$, respectively. Setting $Q=\{x \in D \mid x=A x\}$, we know that $Q$ is nonempty. Set $G_{1}=\{x \in D \mid x \leq A x, x \leq u, \forall u \in Q\}$. It is obvious that $A u_{0} \in G_{1}$ and $G_{1} \neq \emptyset$. Letting $M_{1}$ be a complete partial ordering set of $G_{1}$ and using the same argument as the above, we can find a $\bar{z}$ such that $\bar{z} \in D$ and $\bar{z} \leq A \bar{z}$. Since $\left\{x_{n}\right\} \subset M_{1} \subset G_{1}$, for $\forall u \in Q$ we have $x_{n} \leq u$ and

$$
z_{n}=A x_{n} \leq A u, \quad \bar{z} \leq A u, \quad \bar{z} \leq u .
$$


So $\bar{z}$ is a supper bound of $M_{1}$ in $G_{1}$. According to Zorn's Lemma, there exists a $w \in G_{1}$ such that we have $u^{\prime} \leq w$ for $\forall u^{\prime} \in G_{1}$. Apparently, $w=A w$ and $w$ is a minimal fixed point of $A$. Using an analogous argument, we can obtain that there exists a maximal fixed point of $A$.

REMARK 2.7. Under the assumptions of Theorem 2.6, we can not conclude that there exists only one fixed point of $A$. The feature of this theorem lies in the fact that unlike the classical results in [20-22] we do not need the continuity of $A$.

In order to prove the Ascoli-Arzela's Theorem on time scales in Banach spaces, we introduce the following definition and theorem.

Definition 2.8. [20] Assume that $S$ is a bounded set in E. We define $\alpha(S)=\inf \left\{\delta>0 \mid \begin{array}{c}S \text { can be expressed as the union } S=\cup_{i=1}^{m} S_{i} \\ \text { of a finite number of sets } S_{i} \text { with diameter } \operatorname{diam}\left(S_{i}\right) \leq \delta\end{array}\right\}$.

TheOREM 2.9. [20] Let $S$ be a bounded set of $E$, then $\alpha(S)=0 \Leftrightarrow S$ is a relatively compact set.

\section{Some Definitions and Theorems on Time Scales}

In this section, first we introduce some definitions concerning the calculus on time scales, which can be found in references $[\mathbf{2 4}, \mathbf{2 5}]$. Then we present some new definitions and results in Banach spaces on time scales.

Definition 3.1. $[\mathbf{2 4 , 2 5}]$ A time scale $\mathbb{T}$ is a nonempty closed subset of $\mathbb{R}$. It follows that the jump operators $\sigma, \rho: \mathbb{T} \rightarrow \mathbb{T}$ defined by $\sigma(t)=\inf \{\tau \in \mathbb{T}: \tau>t\}$ and $\rho(t)=\sup \{\tau \in \mathbb{T}: \tau<t\} \quad$ (supplemented by $\inf \emptyset:=\sup \mathbb{T}$ and $\sup \emptyset:=\inf \mathbb{T}$ ) are well defined. The point $t \in \mathbb{T}$ is left-dense, left-scattered, right-dense, rightscattered if $\rho(t)=t, \rho(t)<t, \sigma(t)=t$ and $\sigma(t)>t$, respectively. If $\mathbb{T}$ has a right-scattered minimum $m$, we define $\mathbb{T}_{\kappa}=\mathbb{T}-\{m\}$; otherwise, we set $\mathbb{T}_{\kappa}=\mathbb{T}$. If $\mathbb{T}$ has a left-scattered maximum $M$, we define $\mathbb{T}^{\kappa}=\mathbb{T}-\{M\}$; otherwise, we set $\mathbb{T}^{\kappa}=\mathbb{T}$. The forward graininess is $\mu(t):=\sigma(t)-t$. Similarly, the backward graininess is $\nu(t):=t-\rho(t)$.

Similar to Definition 1.10 in [25], we can define the following delta (nabla) derivative of $x: \mathbb{T} \rightarrow E$ at the point $t \in \mathbb{T}^{\kappa}\left(t \in \mathbb{T}_{\kappa}\right)$.

Definition 3.2. The operator $x: \mathbb{T} \rightarrow E$ is called an abstract function on time scales.

DeFinition 3.3. Let $x: \mathbb{T} \rightarrow E$ be an abstract function and $t \in \mathbb{T}^{\kappa}$. We define the delta derivative of $x(t)$, denoted by $x^{\Delta}(t)$, to be the value $z_{0} \in E$ (provided it exists), with the property that, for any $\varepsilon>0$, there is a neighborhood $U$ of $t$ such that $\left\|\frac{[x(\sigma(t))-x(s)]-x^{\Delta}(t)[\sigma(t)-s]}{\sigma(t)-s}\right\|_{E}<\varepsilon$ for all $s \in U$. For $x: \mathbb{T} \rightarrow E$ and $t \in \mathbb{T}_{\kappa}$, we define the nabla derivative of $x(t)$, denoted by $x^{\nabla}(t)$, to be the value $z_{1} \in E$ (provided it exists), with the property that, for any $\varepsilon>0$, there is a neighborhood $V$ of $t$ such that $\left\|\frac{[x(\rho(t))-x(s)]-x^{\nabla}(t)[\rho(t)-s]}{\rho(t)-s}\right\|_{E}<\varepsilon$ for all $s \in V$.

In order to describe abstract functions that are integrable in the Banach space $E$ on time scales, we use the following notations: the set of the function that $x$ : $\mathbb{T} \rightarrow E$ is continuous, is denoted by $C(\mathbb{T}, E)$. The set of the function $x: \mathbb{T} \rightarrow E$ 
that is differentiable and whose delta (nabla) derivative is continuous, is denoted by $C_{r d}^{\Delta}(\mathbb{T}, E)\left(C_{r d}^{\nabla}(\mathbb{T}, E)\right)$.

REMARK 3.4. The function $x: \mathbb{T} \rightarrow E$ is continuous if it is continuous as a map of the topological space $\mathbb{T}$ into the topological space $E$.

A simpler alternative approach is to construct the integral directly through the standard Lebesgue integration. We can give the following definition which is motivated by $[\mathbf{2 6}]$.

Definition 3.5. Letting $a:=\inf \{s: s \in \mathbb{T}\}$ and $b:=\sup \{s: s \in \mathbb{T}\}$, we define an abstract function $\Psi:[a, b] \rightarrow E$ by

$$
\Psi(t):=\sup \{s \in \mathbb{T}: s \leq t\} \text { for } t \in[a, b] .
$$

Suppose that $f: \mathbb{T}^{\kappa} \rightarrow E$ is an arbitrary abstract function on $\mathbb{T}^{\kappa}$. Then the abstract function $f \circ \Psi:[a, b) \rightarrow E$ is an extension of $f$ to $[a, b)$, and is a constant on "gap" in $\mathbb{T}^{\kappa}$, with the constant equal to the value of $f$ at the left-hand end of the gap. Suppose that $f: \mathbb{T}^{\kappa} \rightarrow \mathbb{R}$ is an arbitrary function on $\mathbb{T}^{\kappa}$, if $f \circ \Psi$ is integrable on the real interval $[a, b)$ in the Lebesgue sense, then we say that $f$ is integrable.

Let $L^{1}(\mathbb{T})$ denote the set of such integrable functions on $\mathbb{T}$. For any $f \in L^{1}(\mathbb{T})$, we define the integral of $f$ as

$$
\int_{s}^{t} f \Delta \tau:=\int_{s}^{t} f \circ \Psi d \tau \text { for } s, t \in \mathbb{T},
$$

with the norm defined by

$$
\|f\|_{L^{1}(\mathbb{T})}=\int_{a}^{b}|f| \Delta t \text { for } f \in L^{1}(\mathbb{T}) .
$$

Definition 3.6. Assume that $f(t): \mathbb{T} \rightarrow E$ is an abstract function and $b, t \in$ $\mathbb{T}$. If $F^{\Delta}(t): \mathbb{T}^{\kappa} \rightarrow E$ and $F^{\Delta}(t)=f(t)$, then we define the delta integral by $\int_{b}^{t} f(s) \Delta s=F(t)-F(b)$. If $F^{\nabla}(t): \mathbb{T}_{\kappa} \rightarrow E$ and $F^{\nabla}(t)=f(t)$, then we define the nabla integral by $\int_{b}^{t} f(s) \nabla s=F(t)-F(b)$.

The following theorem is essential to prove the Ascoli-Arzela's Theorem on time scales. It can be proved by using a similar argument as that of Theorem 1.2.4 in $[\mathbf{2 0}]$.

Theorem 3.7. Assume that $H \subset C\left([a, b]_{\mathbb{T}}, E\right)$ is bounded and equicontinuous, then the following statements are true:

(i) $\alpha_{c}(H)=\alpha\left(H\left([a, b]_{\mathbb{T}}\right)\right)$, where $\alpha_{c}$ denotes the noncompactness measure in $C\left([a, b]_{\mathbb{T}}, E\right)$

(ii) $\alpha\left(H\left([a, b]_{\mathbb{T}}\right)\right)=\max _{t \in[a, b]_{\mathbb{T}}} \alpha(H(t))$.

Now we are ready to introduce and prove the Ascoli-Arzela's Theorem on time scales.

THEOREM 3.8. $H \subset C\left([a, b]_{\mathbb{T}}, E\right)$ is relatively compact if and only if $H$ is equicontinuous and for each $t \in[a, b]_{\mathbb{T}}, H(t)$ is relatively compact set in $E$.

Proof of Theorem 3.8. Assume that $H$ is relatively compact in $C\left([a, b]_{\mathbb{T}}, E\right)$. Clearly, $H(t)$ is a relatively compact set in $E$ for each $t \in[a, b]_{\mathbb{T}}$. According to the Hausdorff theorem, for any $\varepsilon>0$, there is a finite subset $H_{0}=\left\{x_{1}, x_{2}, \ldots, x_{m}\right\} \subset H$ such that there exists a $x_{i} \in H_{0}$ which satisfies

$$
\left\|x-x_{i}\right\|_{E}<\varepsilon \text { for } x \in H .
$$


It is easy to see that $x_{i}(t)(i=1,2, \ldots, m)$ are uniformly continuous on $[a, b]_{\mathbb{T}}$. Hence, there exists a $\delta>0$ such that

$$
\left\|x_{i}(t)-x_{i}\left(t^{\prime}\right)\right\|<\varepsilon \text { whenever }\left|t-t^{\prime}\right|<\delta,
$$

where $i=1,2, \ldots, m$. For any $x \in H$, if $t, t^{\prime} \in[a, b]_{\mathbb{T}}$ satisfy $\left|t-t^{\prime}\right|<\delta$, we choose $x_{i} \in H_{0}$ such that (3.1) holds, then we have

$$
\begin{aligned}
\left\|x(t)-x\left(t^{\prime}\right)\right\| & \leq\left\|x(t)-x_{i}(t)\right\|+\left\|x_{i}(t)-x_{i}\left(t^{\prime}\right)\right\|+\left\|x_{i}\left(t^{\prime}\right)-x\left(t^{\prime}\right)\right\|, \\
& \leq 2\left\|x-x_{i}\right\|_{E}+\left\|x_{i}\left(t^{\prime}\right)-x_{i}\left(t^{\prime}\right)\right\|<3 \varepsilon,
\end{aligned}
$$

which implies that $H$ is equicontinuous.

Conversely, if $H$ is equicontinuous and $H(t)$ is a relatively compact set in $E$ for each $t \in[a, b]_{\mathbb{T}}$, it is easy to show that $H(t)$ is a bounded set in $C\left([a, b]_{\mathbb{T}}, E\right)$. According to Theorem 2.9, we have $\alpha(H(t))=0$ for all $t \in[a, b]_{\mathbb{T}}$. Then it follows from Theorem 3.7 that

$$
\alpha_{c}(H)=\max _{\left.t \in[a, b]_{\mathbb{T}}\right)} \alpha(H(t))=\alpha\left(H\left([a, b]_{\mathbb{T}}\right)\right)=0 .
$$

By applying Theorem 2.9 again, $H$ is relatively compact in $C\left([a, b]_{\mathbb{T}}, E\right)$. Consequently, the proof is completed.

Similar to definitions 1.8 and 1.10 in [24], we can give the following definitions on abstract functions.

DEFINITION 3.9. We say that an abstract function $f: \mathbb{T} \rightarrow E$ is right-increasing at a point $t_{0} \in \mathbb{T} \backslash\{\max \mathbb{T}\}(\max \mathbb{T}$ is the maximum value in $\mathbb{T}$ ) provided that

(i): if $t_{0}$ is right-scattered, then $f\left(\sigma\left(t_{0}\right)\right)-f\left(t_{0}\right)>\theta$;

(ii): if $t_{0}$ is right-dense, then there is a neighborhood $U$ of $t_{0}$ such that $f(t)-$ $f\left(t_{0}\right)>\theta$ for all $t \in U$ with $t>t_{0}$.

Similarly, we say that $f$ is right-decreasing if $f\left(\sigma\left(t_{0}\right)\right)-f\left(t_{0}\right)<\theta$ in (i) and $f(t)-$ $f\left(t_{0}\right)<\theta$ in (ii).

DeFinition 3.10. We say that an abstract function $f: \mathbb{T} \rightarrow E$ attains its local right maximum at point $t_{0} \in \mathbb{T} \backslash\{\max \mathbb{T}\}$ provided that

(i): if $t_{0}$ is right-scattered, then $f\left(\sigma\left(t_{0}\right)\right)-f\left(t_{0}\right) \leq \theta$;

(ii): if $t_{0}$ is right-dense, then there is a neighborhood $U$ of $t_{0}$ such that $f(t)-$ $f\left(t_{0}\right) \leq \theta$ for all $t \in U$ with $t>t_{0}$.

Similarly, we say that $f$ attains its local right minimum if $f\left(\sigma\left(t_{0}\right)\right)-f\left(t_{0}\right) \geq \theta$ in (i) and $f(t)-f\left(t_{0}\right) \geq \theta$ in (ii).

By using the closely similar proofs as that of Theorems 1.9, 1.11 and 1.12 in [24], we can obtain the following Theorems on abstract functions on time scales. We will use these theorems in the proof of our main result in the next section.

TheOREM 3.11. Suppose that the abstract function $f: \mathbb{T} \rightarrow E$ is differential at a point $t_{0} \in \mathbb{T} \backslash\{\max \mathbb{T}\}$. If $f^{\Delta}\left(t_{0}\right)>\theta$, then $f$ is right-increasing. If $f^{\Delta}\left(t_{0}\right)<\theta$, then $f$ is right-decreasing.

TheOrem 3.12. Suppose that the abstract function $f: \mathbb{T} \rightarrow E$ is differential at a point $t_{0} \in \mathbb{T} \backslash\{\max \mathbb{T}\}$. If $f^{\Delta}\left(t_{0}\right)>\theta$, then $f$ attains a local right-minimum at $t_{0}$. If $f^{\Delta}\left(t_{0}\right)<\theta$, then $f$ attains a local right-maximum at $t_{0}$. 
THEOREM 3.13. Suppose that the abstract function $f: \mathbb{T} \rightarrow E$ is differential at a point $t_{0} \in \mathbb{T} \backslash\{\max \mathbb{T}\}$. If $f$ attains a local right-minimum at $t_{0}$, then $f^{\Delta}\left(t_{0}\right) \geq \theta$. If $f$ attains a local right-maximum at $t_{0}$, then $f^{\Delta}\left(t_{0}\right) \leq \theta$.

\section{Existence of Positive Solutions}

Let $E_{1}$ be a compact real Banach space and define the norm as $\|\cdot\|_{E_{1}}$. Let $X=C\left([0, T]_{\mathbb{T}}, E_{1}\right) \cap C^{\Delta}\left((0, T]_{\mathbb{T}^{\kappa}}, E_{1}\right) \cap \ldots \cap C^{\Delta^{n}}\left((0, T]_{\mathbb{T}^{\kappa}}, E_{1}\right)$, and define the norm as

$$
\|u\|_{X}=\max \left\{\|u(t)\|_{E_{1}},\left\|u^{\Delta}(t)\right\|_{E_{1}}, \ldots,\left\|u^{\Delta^{n}}(t)\right\|_{E_{1}}\right\},
$$

where $u \in X$, then $X$ is a Banach space.

Define $P_{1}=\{u \in X \mid u \geq \theta\}$, then $P_{1}$ is a cone in $X$. The partial ordering $\leq$ in $X$ can be defined as: for $\forall v, w \in E_{1}, v \leq w$ if $w-v \in P_{1}$.

To demonstrate the existence of positive solutions to the problem (1.3)-(1.4), our idea is to approximate the singular problem by means of a sequence of nonsingular problems and by using the lower and upper solutions of the nonsingular problem in conjunction with Theorem 2.6, then we establish the existence of solutions to each approximating problem.

Now we state our main result as follows:

THEOREM 4.1. Let $n_{0} \in\{1,2, \ldots\}$ be fixed. Assume that (H1)-(H3) hold and the following conditions are satisfied:

(A1): let $e$ be the unit element in the Banach space $E_{1}$. For each $n \in$ $\left\{n_{0}, n_{0}+1, \ldots\right\} \equiv \mathbb{N}_{1}$, there exists a sequence $\left\{\rho_{n} e\right\} \subset X$ such that $\left\{\rho_{n}\right\}$ is a strictly monotone decreasing sequence with $\lim _{n \rightarrow \infty} \rho_{n}=0$, and

$$
q(t) f\left(t, \rho_{n} e, \theta, \ldots, \theta\right) \geq \theta \text { for } t \in\left[\frac{1}{2^{n+1}}, T-\frac{1}{2^{n+1}}\right]_{\mathbb{T}},
$$

where $\rho_{n} \in \mathbb{R}$;

(A2): there exists a function $\alpha(t) \in X$ and $\varphi_{p}\left(\alpha^{\Delta}(t)\right) \in C^{\nabla}\left((0, T)_{\mathbb{T}}, E_{1}\right)$ such that $\alpha(0)=\theta, \alpha(t)>\theta$ on $(0, T]_{\mathbb{T}}$, and

$$
\sum_{j=1}^{m_{1}} \phi_{j}\left(\alpha\left(\xi_{j}^{*}\right)\right)-\sum_{i=1}^{m_{2}} \psi_{i}\left(\alpha^{\Delta}\left(\xi_{i}\right)\right) \leq 0
$$

together with $\left(\varphi_{p}\left(\alpha^{\Delta}(t)\right)\right)^{\nabla}+q(t) f\left(t, \alpha(t), \alpha^{\Delta}(t), \ldots, \alpha^{\Delta^{n}}(t)\right) \geq \theta$ for $t \in$ $(0, T)_{\mathbb{T}}$

(A3): there exists a function $\beta(t) \in X$ and $\varphi_{p}\left(\beta^{\Delta}(t)\right) \in C^{\nabla}\left((0, T)_{\mathbb{T}}, E_{1}\right)$ such that

$$
\begin{aligned}
& \beta(t) \geq \alpha(t) \text { and } \beta(t) \geq \rho_{n_{0}} \text { e for } t \in[0, T]_{\mathbb{T}}, \sum_{j=1}^{m_{1}} \phi_{j}\left(\beta\left(\xi_{j}^{*}\right)\right)-\sum_{i=1}^{m_{2}} \psi_{i}\left(\beta^{\Delta}\left(\xi_{i}\right)\right)>0, \\
& \left(\varphi_{p}\left(\beta^{\Delta}(t)\right)\right)^{\nabla}+q(t) f\left(t, \beta(t), \beta^{\Delta}(t), \ldots, \beta^{\Delta^{n}}(t)\right) \leq \theta \text { for } t \in\left[\frac{1}{2^{n_{0}+1}}, T-\frac{1}{2^{n_{0}+1}}\right]_{\mathbb{T}}, \\
& \left(\varphi_{p}\left(\beta^{\Delta}(t)\right)\right)^{\nabla}+q(t) f\left(\frac{1}{2^{n_{0}+1}}, \beta(t), \beta^{\Delta}(t), \ldots, \beta^{\Delta^{n}}(t)\right) \leq \theta \text { for } t \in\left(0, \frac{1}{2^{n_{0}+1}}\right)_{\mathbb{T}}, \\
& \quad \text { and }
\end{aligned}
$$

$$
\begin{aligned}
& \left(\varphi_{p}\left(\beta^{\Delta}(t)\right)\right)^{\nabla}+q(t) f\left(T-\frac{1}{2^{n_{0}+1}}, \beta(t), \beta^{\Delta}(t), \ldots, \beta^{\Delta^{n}}(t)\right) \\
& \leq \theta \text { for } t \in\left(T-\frac{1}{2^{n_{0}+1}}, T\right)_{\mathbb{T}} .
\end{aligned}
$$


Then the BVPs (1.3)-(1.4) has at least a positive solution $u(t) \in X$ such that

$$
\varphi_{p}\left(u^{\Delta}(t)\right) \in C^{\nabla}\left((0, T)_{\mathbb{T}}, E_{1}\right) \text { with } u(t) \geq \alpha(t) \text { for } t \in[0, T]_{\mathbb{T}} .
$$

Proof of TheOrem 4.1. Since the cone $P_{1}=\{u \in X \mid u \geq \theta\}$ induces the partial ordering $\leq$ in $X$. Let $\xi=\min \left\{\xi_{1}, \xi_{1}^{*}\right\}$. Without loss of generality, fixing $n \in \mathbb{N}_{1}$, we suppose that $\inf _{t \in[\xi, T]_{\mathbb{T}}} \alpha(t) \geq \rho_{n} e$. Let $t_{n} \in(0, \xi)_{\mathbb{T}}$ satisfy

$$
\alpha\left(t_{n}\right)=\rho_{n} e \text { and } \alpha(t) \leq \rho_{n} e \text { for } t \in\left[0, t_{n}\right]_{\mathbb{T}},
$$

and

$$
\alpha_{n}(t)=\left\{\begin{array}{ll}
\rho_{n} e & \text { if } t \in\left[0, t_{n}\right]_{\mathbb{T}}, \\
\alpha(t) & \text { if } t \in\left[t_{n}, T\right]_{\mathbb{T}},
\end{array} \quad \text { here } \alpha\left(t_{n}\right)=\rho_{n} e\right.
$$

Assume that $\omega_{n}=\left[\frac{1}{2^{n+1}}, T-\frac{1}{2^{n+1}}\right]_{\mathbb{T}}$, then we have

$$
\omega_{n}(t)= \begin{cases}\max \left\{\frac{1}{2^{n+1}}, t\right\}, & \text { for } t \in\left[0, T-\frac{1}{2^{n+1}}\right]_{\mathbb{T}}, \\ \min \left\{T-\frac{1}{2^{n+1}}, t\right\}, & \text { for } t \in\left[T-\frac{1}{2^{n+1}}, T\right]_{\mathbb{T}},\end{cases}
$$

and

$$
f_{n}\left(t, x_{0}, x_{1}, \ldots, x_{n}\right)=\max \left\{f\left(t, x_{0}, x_{1}, \ldots, x_{n}\right), f\left(\omega_{n}(t), x_{0}, x_{1}, \ldots, x_{n}\right)\right\} .
$$

We define a sequence $h_{n_{0}}\left(t, x_{0}, x_{1}, \ldots, x_{n}\right)=f_{n_{0}}\left(t, x_{0}, x_{1}, \ldots, x_{n}\right)$ and

$$
h_{n}\left(t, x_{0}, x_{1}, \ldots, x_{n}\right)=\min \left\{f_{n_{0}}\left(t, x_{0}, x_{1}, \ldots, x_{n}\right), \ldots, f_{n}\left(t, x_{0}, x_{1}, \ldots, x_{n}\right)\right\},
$$

where $n=n_{0}+1, n_{0}+2, \ldots$

Then, for $\left(t, x_{0}, x_{1}, \ldots, x_{n}\right) \in(0, T)_{\mathbb{T}} \times P_{1} \times E_{1}^{n}$ we have

$$
\begin{aligned}
f\left(t, x_{0}, x_{1}, \ldots, x_{n}\right) & \leq \ldots \leq h_{n+1}\left(t, x_{0}, x_{1}, \ldots, x_{n}\right), \\
& \leq h_{n}\left(t, x_{0}, x_{1}, \ldots, x_{n}\right), \\
& \leq \ldots \leq h_{n_{0}}\left(t, x_{0}, x_{1}, \ldots, x_{n}\right) .
\end{aligned}
$$

For $\left(t, x_{0}, x_{1}, \ldots, x_{n}\right) \in(0, T)_{\mathbb{T}} \times P_{1} \times E_{1}^{n}$ we get

$$
h_{n}\left(t, x_{0}, x_{1}, \ldots, x_{n}\right)=f\left(t, x_{0}, x_{1}, \ldots, x_{n}\right) .
$$

Consider the BVPs

$$
\begin{gathered}
\left(\varphi_{p}\left(u_{n_{0}}^{\Delta}(t)\right)\right)^{\nabla}+q(t) h_{n_{0}}^{*}\left(t, u_{n_{0}}(t), u_{n_{0}}^{\Delta}(t), \ldots, u_{n_{0}}^{\Delta^{n}}(t)\right)=\theta, t \in(0, T)_{\mathbb{T}} \\
u(\theta)=\rho_{n_{0}} e, \sum_{j=1}^{m_{1}} \phi_{j}^{*}\left(u_{n_{0}}\left(\xi_{j}^{*}\right)\right)-\sum_{i=1}^{m_{2}} \psi_{i}^{*}\left(u_{n_{0}}^{\Delta}\left(\xi_{i}\right)\right)=\rho_{n_{0}}
\end{gathered}
$$

where

$$
\begin{gathered}
h_{n_{0}}^{*}= \begin{cases}h_{n_{0}}\left(t, \alpha_{n_{0}}(t), \alpha_{n_{0}}^{\Delta}(t), \ldots, \alpha_{n_{0}}^{\Delta^{n}}(t)\right)+r\left(\alpha_{n_{0}}(t)-u_{n_{0}}(t)\right), & u(t) \leq \alpha_{n_{0}}(t), \\
h_{n_{0}}\left(t, u_{n_{0}}(t), u_{n_{0}}^{\Delta}(t), \ldots, u_{n_{0}}^{\Delta^{n}}(t)\right), \alpha_{n_{0}}(t) \leq u_{n_{0}}(t) \leq \beta(t), & \\
h_{n_{0}}\left(t, \beta(t), \beta^{\Delta}(t), \ldots, \beta^{\Delta^{n}}(t)\right)+r\left(\beta(t)-u_{n_{0}}(t)\right), & u_{n_{0}}(t) \geq \beta(t),\end{cases} \\
\phi_{j}^{*}\left(z_{j}^{*}\right)= \begin{cases}\phi_{j}\left(\alpha\left(\xi_{j}^{*}\right)\right), & z_{j}^{*} \leq \alpha_{n_{0}}\left(\xi_{j}^{*}\right)=\alpha\left(\xi_{j}^{*}\right), \\
\phi_{j}\left(z_{j}^{*}\right), & \alpha_{n_{0}}\left(\xi_{j}^{*}\right) \leq z_{j}^{*} \leq \beta\left(\xi_{j}^{*}\right), \quad j=1, \ldots, m_{1}-1, \\
\phi_{j}\left(\beta\left(\xi_{j}^{*}\right)\right), & z_{j}^{*} \geq \beta\left(\xi_{j}^{*}\right),\end{cases}
\end{gathered}
$$

and

$$
\psi_{i}^{*}\left(z_{i}\right)= \begin{cases}\psi_{i}\left(\alpha^{\Delta}\left(\xi_{i}\right)\right), & z_{i} \leq \alpha_{n_{0}}^{\Delta}\left(\xi_{i}\right)=\alpha^{\Delta}\left(\xi_{i}\right), \\ \psi_{i}\left(z_{i}\right), & \alpha_{n_{0}}^{\Delta}\left(\xi_{i}\right) \leq z_{i} \leq \beta^{\Delta}\left(\xi_{i}\right), \quad i=1, \ldots, m_{2} \\ \psi_{i}\left(\beta^{\Delta}\left(\xi_{i}\right)\right), & z_{i} \geq \beta^{\Delta}\left(\xi_{i}\right) .\end{cases}
$$


Let $r(u): E_{1} \rightarrow B_{E_{1}}(\theta, 1)=\left\{x: x \in E_{1}\right.$ and $\left.\|x\|_{E_{1}} \leq 1\right\}$ be the radial retraction function defined by

$$
r(u)= \begin{cases}u, & \|u\|_{E_{1}} \leq 1 \\ \frac{u}{\|u\|_{E_{1}}}, & \|u\|_{E_{1}}>1 .\end{cases}
$$

Assume that

$$
C_{\theta}^{\Delta^{n}}\left([0, T]_{\mathbb{T}^{\kappa n}}, E_{1}\right)=\{u \in X: u(0)=\theta\}
$$

and

$$
C_{\rho_{n_{0}}}^{\Delta^{n}}\left([0, T]_{\mathbb{T}^{\kappa} n}, E_{1}\right)=\left\{u \in X: u(0)=\rho_{n_{0}} e\right\} .
$$

We define the mappings $L_{p}, F: C_{\rho_{n_{0}}}^{\Delta^{n}}\left([0, T]_{\mathbb{T}^{\kappa n}}, E_{1}\right) \rightarrow C_{\theta}^{\Delta^{n}}\left([0, T]_{\mathbb{T}^{\kappa n}}, E_{1}\right)$ as

$$
L_{p} u(t)=\left(\varphi_{p}\left(u^{\Delta}(t)\right)-\varphi_{p}\left(u^{\Delta}(0)\right), \sum_{j=1}^{m_{1}} \phi_{j}^{*}\left(u\left(\xi_{j}^{*}\right)\right),\right.
$$

and

$$
F u(t)=\left(-\int_{0}^{t} q(x) h_{n_{0}}^{*}\left(x, u(x), u^{\Delta}(x), \ldots, u^{\Delta^{n}}(x)\right) \nabla x, \sum_{i=1}^{m_{2}} \psi_{i}^{*}\left(u^{\Delta}\left(\xi_{i}\right)\right)+\rho_{n_{0}}\right) .
$$

Moreover, if

$$
L_{p} v=(u, \gamma) \text { for } u \in C_{\theta}^{\Delta^{n}}\left([0, T]_{\mathbb{T}^{\kappa} n}, E_{1}\right),
$$

then we have

$$
\gamma=\sum_{i=1}^{m_{1}} \phi_{j}^{*}\left(\rho_{n_{0}}+\int_{0}^{\xi_{j}^{*}} \varphi_{q}\left(u(x)-\sum_{j=1}^{m_{1}} \phi_{j}^{*}\left(u\left(\xi_{j}^{*}\right)\right)\right) \Delta x\right) .
$$

This yields $v(t)=\rho_{n_{0}}+\int_{0}^{t} \varphi_{q}\left(u(x)-\sum_{j=1}^{m_{1}} \phi_{j}^{*}\left(u\left(\xi_{j}^{*}\right)\right)\right) \Delta x$. Hence $L_{p}^{-1}$ exists. It is clear that solving the BVPs (4.3)-(4.4) is equivalent to finding a fixed point of $u_{n_{0}}=$ $L_{p}^{-1} F u_{n_{0}} \equiv N u_{n_{0}}$, where $N=L_{p}^{-1} F: C_{\rho_{n_{0}}}^{\Delta^{n}}\left([0, T]_{\mathbb{T}^{\kappa} n}, E_{1}\right) \rightarrow C_{\rho_{n_{0}}}^{\Delta^{n}}\left([0, T]_{\mathbb{T}^{\kappa} n}, E_{1}\right)$ is monotone because of inequality (4.2). In order to apply Theorem 2.6, we only need to prove that conditions $($ ii) and (iii) of Theorem 2.6 hold.

For $\forall t \in[0, T]_{\mathbb{T}^{\kappa}}$, the bounded set $B$ in $C_{\rho_{n_{0}}}^{\nabla^{n}}\left((0, T)_{\mathbb{T}}, E_{1}\right)$, if $u(t) \in B$, we deduce that

$$
\sup _{t}\left\|L_{p}^{-1} F u(t)\right\|_{E_{1}} \leq \sup \left\|L_{p}^{-1} F\right\|\|u\|_{E_{1}}<\infty .
$$

For $\forall t_{1}, t_{2} \in[0, T]_{\mathbb{T}^{k}}$, we obtain that

$$
\begin{aligned}
& \left\|L_{p}^{-1} F u\left(t_{1}\right)-L_{p}^{-1} F u\left(t_{2}\right)\right\|_{E_{1}} \\
& =\left\|L_{p}^{-1}\left(\int_{t_{1}}^{t_{2}} q(x) h_{n_{0}}^{*}\left(x, u(x), u^{\Delta}(x), \ldots, u^{\Delta^{n}}(x)\right) \Delta x\right)\right\|_{E_{1}}, \\
& \leq \sup \left\|L_{p}^{-1}\right\| \int_{t_{1}}^{t_{2}}\left\|q(x) h_{n_{0}}^{*}\left(x, u(x), u^{\Delta}(x), \ldots, u^{\Delta^{n}}(x)\right)\right\|_{E_{1}} \Delta x, \\
& \rightarrow 0 \text { as } t_{1} \rightarrow t_{2} .
\end{aligned}
$$

It follows from Theorem 3.8 that $N$ is compact, so the condition ( $i i$ ) holds.

In the following, we shall show that

$$
\alpha_{n_{0}}(t) \leq u_{n_{0}}(t) \text { for } t \in[0, T]_{\mathbb{T}} .
$$

If inequality (4.6) is not true then the abstract function $u_{n_{0}}(t)-\alpha_{n_{0}}(t)$ has a local right minimum at some $\tau \in(0, T]_{\mathbb{T}}$. We consider two cases, that is, $\tau \in(0, T)_{\mathbb{T}}$ and $\tau=T$.

Case I. Assume that $\tau \in(0, T)_{\mathbb{T}}$, then we claim

$$
\left(\varphi_{p}\left(u_{n_{0}}^{\Delta}\right)\right)^{\nabla}(\tau)-\left(\varphi_{p}\left(\alpha_{n_{0}}^{\Delta}\right)\right)^{\nabla}(\tau) \geq \theta
$$


Since the abstract function $u_{n_{0}}(t)-\alpha_{n_{0}}(t)$ has a local right minimum at some $\tau \in(0, T)_{\mathbb{T}}$, in view of Theorems 3.11-3.13, we have $u_{n_{0}}^{\Delta}(\tau)-\alpha_{n_{0}}^{\Delta}(\tau) \geq \theta$ and there exists a $\delta$ with $\tau-\delta \in[0, \tau)_{\mathbb{T}}$ such that $u_{n_{0}}^{\Delta}(t)-\alpha_{n_{0}}^{\Delta}(t) \leq \theta$ for $t \in[\tau-\delta, \tau)_{\mathbb{T}}$. Thus we have

$$
\varphi_{p}\left(u_{n_{0}}^{\Delta}(\tau)\right)-\varphi_{p}\left(\alpha_{n_{0}}^{\Delta}(\tau)\right)-\left(\varphi_{p}\left(u_{n_{0}}^{\Delta}(t)\right)-\varphi_{p}\left(\alpha_{n_{0}}^{\Delta}(t)\right)\right) \geq \theta \text { for } t \in[\tau-\delta, \tau)_{\mathbb{T}},
$$

which leads to

$$
\frac{\varphi_{p}\left(u_{n_{0}}^{\Delta}(t)\right)-\varphi_{p}\left(u_{n_{0}}^{\Delta}(\tau)\right)}{t-\tau} \geq \frac{\varphi_{p}\left(\alpha_{n_{0}}^{\Delta}(t)\right)-\varphi_{p}\left(\alpha_{n_{0}}^{\Delta}(\tau)\right)}{t-\tau} \text { for } t \in[\tau-\delta, \tau)_{\mathbb{T}} .
$$

If $\tau$ is left-dense, for $t \in[\tau-\delta, \tau)$ we derive that

$$
\begin{aligned}
\left(\varphi_{p}\left(u_{n_{0}}^{\Delta}\right)\right)^{\nabla}(\tau) & =\lim _{t \rightarrow \tau} \frac{\varphi_{p}\left(u_{n_{0}}^{\prime}(t)\right)-\varphi_{p}\left(u_{n_{0}}^{\prime}(\tau)\right)}{t-\tau}, \\
& \geq \lim _{t \rightarrow \tau} \frac{\varphi_{p}\left(\alpha_{n_{0}}^{\prime}(t)\right)-\varphi_{p}\left(\alpha_{n_{0}}^{\prime}(\tau)\right)}{t-\tau}, \\
& =\left(\varphi_{p}\left(\alpha_{n_{0}}^{\prime}\right)\right)^{\prime}(\tau)=\left(\varphi_{p}\left(\alpha_{n_{0}}^{\Delta}\right)\right)^{\nabla}(\tau) .
\end{aligned}
$$

If $\tau$ is left-scattered, by virtue of (4.8) we deduce that

$$
\begin{aligned}
\left(\varphi_{p}\left(u_{n_{0}}^{\Delta}\right)\right)^{\nabla}(\tau) & =\frac{\varphi_{p}\left(u_{n_{0}}^{\Delta}(\tau)\right)-\varphi_{p}\left(u_{n_{0}}^{\Delta}(\rho(\tau))\right)}{\tau-\rho(\tau)}, \\
& \geq \frac{\varphi_{p}\left(\alpha_{n_{0}}^{\Delta}(\tau)\right)-\varphi_{p}\left(\alpha_{n_{0}}^{\Delta}(\rho(\tau))\right)}{\tau-\rho(\tau)} \\
& =\left(\varphi_{p}\left(\alpha_{n_{0}}^{\Delta}\right)\right)^{\nabla}(\tau) .
\end{aligned}
$$

Hence, we see that inequality (4.7) is true.

However, by (4.1), (4.5) and $u_{n_{0}}(\tau)-\alpha_{n_{0}}(\tau)<\theta$, we obtain

$$
\begin{aligned}
& \left(\varphi_{p}\left(u_{n_{0}}^{\Delta}(\tau)\right)\right)^{\nabla}-\left(\varphi_{p}\left(\alpha_{n_{0}}^{\Delta}(\tau)\right)\right)^{\nabla} \\
& =-\left[q(\tau) h_{n_{0}}\left(\tau, \alpha_{n_{0}}(\tau), \alpha_{n_{0}}^{\Delta}(\tau), \ldots, \alpha_{n_{0}}^{\Delta^{n}}(\tau)\right)\right. \\
& \left.+q(\tau) r\left(\alpha_{n_{0}}(\tau)-u_{n_{0}}(\tau)\right)+\left(\varphi_{p}\left(\alpha_{n_{0}}^{\Delta}(\tau)\right)\right)^{\nabla}\right], \\
& =\left\{\begin{array}{c}
-\left[q(\tau) h_{n_{0}}\left(\tau, \rho_{n_{0}} e, \theta, \ldots, \theta\right)+q(\tau) r\left(\rho_{n_{0}} e-u_{n_{0}}(\tau)\right)\right], \\
\tau \in\left(0, t_{n_{0}}\right)_{\mathbb{T}}, \\
-q(\tau) h_{n_{0}}\left(\tau, \alpha(\tau), \alpha^{\Delta}(\tau), \ldots, \alpha^{\Delta^{n}}(\tau)\right) \\
-q(\tau) r\left(\alpha(\tau)-u_{n_{0}}(\tau)\right) \\
+\left(\varphi_{p}\left(\alpha^{\Delta}(\tau)\right)\right)^{\nabla}, \tau \in\left[t_{n_{0}}, T\right)_{\mathbb{T}} .
\end{array}\right.
\end{aligned}
$$

Assume that $\tau \in\left[\frac{1}{2^{n_{0}+1}}, T-\frac{1}{2^{n+1}}\right]_{\mathbb{T}}$, then we have

$h_{n_{0}}\left(\tau, x_{0}, x_{1}, \ldots, x_{n}\right)=f\left(\tau, x_{0}, x_{1}, \ldots, x_{n}\right)$, for $\left(x_{0}, x_{1}, \ldots, x_{n}\right) \in P \times E_{1}^{n-1}$.

It follows from (A1), (A2) and $q(\tau)>0$ that

$$
\begin{aligned}
& \left(\varphi_{p}\left(u_{n_{0}}^{\Delta}(\tau)\right)\right)^{\nabla}-\left(\varphi_{p}\left(\alpha_{n_{0}}^{\Delta}(\tau)\right)\right)^{\nabla} \\
& = \begin{cases}-\left[q(\tau) f\left(\tau, \rho_{n_{0}} e, \theta, \ldots, \theta\right)+q(\tau) r\left(\rho_{n_{0}} e-u_{n_{0}}(\tau)\right)\right], & \tau \in\left(0, t_{n_{0}}\right)_{\mathbb{T}}, \\
-q(\tau) f\left(\tau, \alpha(\tau), \alpha^{\Delta}(\tau), \ldots, \alpha^{\Delta^{n}}(\tau)\right) & \tau \in\left[t_{n_{0}}, T\right)_{\mathbb{T}} . \\
-q(\tau) r\left(\alpha(\tau)-u_{n_{0}}(\tau)\right)-\left(\varphi_{p}\left(\alpha^{\Delta}(\tau)\right)\right) \nabla, & \end{cases} \\
& <\theta .
\end{aligned}
$$


This yields a contradiction with (4.7).

Similarly, assume that $\tau \in\left(0, \frac{1}{2^{n} 0+1}\right)_{\mathbb{T}} \cup\left(T-\frac{1}{2^{n} 0+1}, T\right)_{\mathbb{T}}$, then we have

$$
\begin{aligned}
& h_{n_{0}}\left(\tau, x_{0}, x_{1}, \ldots, x_{n}\right) \\
= & f_{n_{0}}\left(\tau, x_{0}, x_{1}, \ldots, x_{n}\right), \\
= & \max \left\{f\left(\tau, x_{0}, x_{1}, \ldots, x_{n}\right), f\left(\omega_{n_{0}}(\tau), x_{0}, x_{1}, \ldots, x_{n}\right)\right\}, \\
= & \max \left\{f\left(\frac{1}{2^{n_{0}+1}}, x_{0}, x_{1}, \ldots, x_{n}\right),\right. \\
& \left.f\left(T-\frac{1}{2^{n_{0}+1}}, x_{0}, x_{1}, \ldots, x_{n}\right), f\left(\tau, x_{0}, x_{1}, \ldots, x_{n}\right)\right\} .
\end{aligned}
$$

In view of $(\mathrm{A} 1),(\mathrm{A} 2)$ and $q(\tau)>0$, we deduce that

$$
\begin{aligned}
& \left(\varphi_{p}\left(u_{n_{0}}^{\Delta}(\tau)\right)\right)^{\nabla}-\left(\varphi_{p}\left(\alpha_{n_{0}}^{\Delta}(\tau)\right)\right)^{\nabla} \\
& \leq\left\{\begin{array}{l}
-\left[q(\tau) f\left(\frac{1}{2^{n_{0}+1}}, \rho_{n_{0}} e, \theta, \ldots, \theta\right)+q(\tau) r\left(\rho_{n_{0}} e-u_{n_{0}}(\tau)\right)\right], \tau \in\left(0, t_{n_{0}}\right)_{\mathbb{T}}, \\
-\left[q(\tau) f\left(\tau, \alpha(\tau), \alpha^{\Delta}(\tau), \ldots, \alpha^{\Delta^{n}}(\tau)\right)+\left(\varphi_{p}\left(\alpha^{\Delta}(\tau)\right)\right)^{\nabla}\right] \\
-q(\tau) r\left(\alpha(\tau)-u_{n_{0}}(\tau)\right), \tau \in\left[t_{n_{0}}, T-\frac{1}{2^{n_{0}+1}}\right)_{\mathbb{T}}, \\
-\left[q(\tau) f\left(T-\frac{1}{2^{n_{0}+1}}, \rho_{n_{0}} e, \theta, \ldots, \theta\right)+q(\tau) r\left(\rho_{n_{0}} e-u_{n_{0}}(\tau)\right)\right], \\
\tau \in\left[T-\frac{1}{2^{n_{0}+1}}, T\right)_{\mathbb{T}},
\end{array}\right. \\
& <\theta .
\end{aligned}
$$

This gives another contradiction with (4.7).

Case II. Assume that $\tau=T$. It gives, $\alpha_{n_{0}}(T)-u_{n_{0}}(T)>\theta$, which implies $\phi_{m_{1}}\left(\alpha_{n_{0}}(T)\right)-\phi_{m_{1}}\left(u_{n_{0}}(T)\right)>0$. By using the closely similar discussion as that used in $[\mathbf{1 0}]$ we can obtain the same contradiction.

Thus, in cases I and II inequality (4.6) is true. In particular, since $\alpha(t) \leq \alpha_{n_{0}}(t)$, for $t \in[0, T]_{\mathbb{T}}$ we obtain $\alpha(t) \leq \alpha_{n_{0}}(t) \leq u_{n_{0}}(t)$ for $t \in[0, T]_{\mathbb{T}}$. Essentially, by the same discussion as that for inequality (4.6) we have $u_{n_{0}}(t) \leq \beta(t)$ for $t \in[0, T]_{\mathbb{T}}$. Hence, we obtain that

$$
\alpha(t) \leq \alpha_{n_{0}}(t) \leq u_{n_{0}}(t) \leq \beta(t) \text { for } t \in[0, T]_{\mathbb{T}} .
$$

Now, we discuss the BVPs

$$
\begin{gathered}
\left(\varphi_{p}\left(u_{n_{0}+1}^{\Delta}(t)\right)\right)^{\nabla}+q(t) h_{n_{0}+1}^{*}\left(t, u_{n_{0}+1}(t), u_{n_{0}+1}^{\Delta}(t), \ldots, u_{n_{0}+1}^{\Delta^{n}}(t)\right)=\theta, t \in(0, T)_{\mathbb{T}}, \\
u_{n_{0}+1}(0)=\rho_{n_{0}+1} e, \quad \sum_{j=1}^{m_{1}} \phi_{j}^{*}\left(u_{n_{0}+1}\left(\xi_{j}^{*}\right)\right)-\sum_{i=1}^{m_{2}} \psi_{i}^{*}\left(u_{n_{0}+1}^{\Delta}\left(\xi_{i}\right)\right)=\rho_{n_{0}+1},
\end{gathered}
$$

where

$$
\begin{aligned}
& h_{n_{0}+1}^{*}\left(t, u_{n_{0}+1}(t), u_{n_{0}+1}^{\Delta}(t), \ldots u_{n_{0}+1}^{\Delta^{n}}(t)\right) \\
& = \begin{cases}h_{n_{0}+1}\left(t, \alpha_{n_{0}+1}(t), \alpha_{n_{0}+1}^{\Delta}(t), \ldots, \alpha_{n_{0}+1}^{\Delta^{n}}(t)\right) \\
+r\left(\alpha_{n_{0}+1}(t)-u_{n_{0}+1}(t)\right), & u(t) \leq \alpha_{n_{0}+1}(t), \\
h_{n_{0}+1}\left(t, u_{n_{0}+1}(t), u_{n_{0}+1}^{\Delta}(t), \ldots u_{n_{0}+1}^{\Delta^{n}}(t)\right), & \alpha_{n_{0}+1}(t) \leq u_{n_{0}+1}(t) \leq u_{n_{0}}(t), \\
h_{n_{0}+1}\left(t, u_{n_{0}}(t), u_{n_{0}}^{\Delta}(t), \ldots, u_{n_{0}}^{\Delta^{n}}(t)\right) & u_{n_{0}+1}(t) \geq u_{n_{0}}(t), \\
+r\left(u_{n_{0}}(t)-u_{n_{0}+1}(t)\right), & \end{cases} \\
& \phi_{j}^{*}\left(z_{j}^{*}\right)= \begin{cases}\phi_{j}\left(\alpha\left(\xi_{j}^{*}\right)\right), & z_{j}^{*} \leq \alpha_{n_{0}+1}\left(\xi_{j}^{*}\right)=\alpha\left(\xi_{j}^{*}\right), \\
\phi_{j}\left(z_{j}^{*}\right), & \alpha_{n_{0}+1}\left(\xi_{j}^{*}\right) \leq z_{j}^{*} \leq u_{n_{0}}\left(\xi_{j}^{*}\right), \quad j=1, \ldots, m_{1}-1 . \\
\phi_{j}\left(u_{n_{0}}\left(\xi_{j}^{\prime}\right)\right), & z_{j}^{*} \geq u_{n_{0}}\left(\xi_{j}^{*}\right),\end{cases}
\end{aligned}
$$


and

$$
\psi_{i}^{*}\left(z_{i}\right)= \begin{cases}\psi_{i}\left(\alpha^{\Delta}\left(\xi_{i}\right)\right), & z_{i} \leq \alpha_{n_{0}+1}^{\Delta}\left(\xi_{i}\right)=\alpha^{\Delta}\left(\xi_{i}\right), \\ \psi_{i}\left(z_{i}\right), & \alpha_{n_{0}+1}^{\Delta}\left(\xi_{i}\right) \leq z_{i} \leq u_{n_{0}}^{\Delta}\left(\xi_{i}\right), \quad i=1, \ldots, m_{2} . \\ \psi_{i}\left(u_{n_{0}}^{\Delta}\left(\xi_{i}\right)\right), & z_{i} \geq u_{n_{0}}^{\Delta}\left(\xi_{i}\right) .\end{cases}
$$

Applying the same argument as that of inequality (4.9), we derive

$$
\alpha(t) \leq \alpha_{n_{0}+1}(t) \leq u_{n_{0}+1}(t) \leq u_{n_{0}}(t) \text { for } t \in[0, T]_{\mathbb{T}} .
$$

Assume that we have $u_{k}(t)$ for some $k \in\left\{n_{0}+1, n_{0}+2, \ldots\right\}$ satisfying

$$
\alpha_{k}(t) \leq u_{k}(t) \leq u_{k-1}(t) \text { for } t \in[0, T]_{\mathbb{T}} .
$$

Then we consider the BVPs

$$
\begin{gathered}
\left(\varphi_{p}\left(u_{k+1}^{\Delta}(t)\right)\right)^{\nabla}+q(t) h_{k+1}^{*}\left(t, u_{k+1}(t), u_{k+1}^{\Delta}(t), \ldots, u_{k+1}^{\Delta^{n}}(t)\right)=\theta, t \in(0, T)_{\mathbb{T}}, \\
u_{k+1}(0)=\rho_{k+1} e, \quad \sum_{j=1}^{m_{1}} \phi_{j}\left(u\left(\xi_{j}^{*}\right)\right)-\sum_{i=1}^{m} \psi_{i}^{*}\left(u_{k+1}^{\Delta}\left(\xi_{i}\right)\right)=\rho_{k+1},
\end{gathered}
$$

where

$$
\begin{aligned}
& h_{k+1}^{*}\left(t, u_{k+1}(t), u_{k+1}^{\Delta}(t), \ldots, u_{k+1}^{\Delta^{n}}(t)\right) \\
& = \begin{cases}h_{k+1}\left(t, \alpha_{k+1}(t), \alpha_{k+1}^{\Delta}(t), \ldots, \alpha_{k+1}^{\Delta^{n}}(t)\right) & \\
+r\left(\alpha_{k+1}(t)-u_{k+1}(t)\right), & u_{k+1}(t) \leq \alpha_{k+1}(t), \\
h_{k+1}\left(t, u_{k+1}(t), u_{k+1}^{\Delta}(t), \ldots, u_{k+1}^{\Delta^{n}}(t)\right), & \alpha_{k+1}(t) \leq u_{k+1}(t) \leq u_{k}(t), \\
h_{k+1}\left(t, u_{k}(t), u_{k}^{\Delta}(t), \ldots, u_{k}^{\Delta^{n}}(t)\right) & \\
+r\left(u_{k}(t)-u_{k+1}(t)\right), & u_{k+1}(t) \geq u_{k}(t),\end{cases} \\
& \phi_{j}^{*}\left(z_{j}^{*}\right)= \begin{cases}\phi_{j}\left(\alpha\left(\xi_{j}^{*}\right)\right), & z_{j}^{*} \leq \alpha_{k+1}\left(\xi_{j}^{*}\right)=\alpha\left(\xi_{j}^{*}\right), \\
\phi_{j}\left(z_{j}^{*}\right), & \alpha_{k+1}\left(\xi_{j}^{*}\right) \leq z_{j}^{*} \leq u_{k}\left(\xi_{j}^{*}\right), \quad j=1, \ldots, m_{1}-1 . \\
\phi_{j}\left(u_{k}\left(\xi_{j}^{*}\right)\right), & z_{j}^{*} \geq u_{k}\left(\xi_{j}^{*}\right),\end{cases}
\end{aligned}
$$

and

$$
\psi_{i}^{*}\left(z_{i}\right)= \begin{cases}\psi_{i}\left(\alpha^{\Delta}\left(\xi_{i}\right)\right), & z_{i} \leq \alpha_{k+1}^{\Delta}\left(\xi_{i}\right)=\alpha^{\Delta}\left(\xi_{i}\right), \\ \psi_{i}\left(z_{i}\right), & \alpha_{k+1}^{\Delta}\left(\xi_{i}\right) \leq z_{i} \leq u_{k}^{\Delta}\left(\xi_{i}\right), \quad i=1, \ldots, m_{2} . \\ \psi_{i}^{\Delta}\left(u_{k}\left(\xi_{i}\right)\right), & z_{i} \geq u_{k}^{\Delta}\left(\xi_{i}\right) .\end{cases}
$$

Again applying the same discussion as that for inequality (4.9) we find

$$
\alpha(t) \leq \alpha_{k+1}(t) \leq u_{k+1}(t) \leq u_{k}(t) \text { for } t \in[0, T]_{\mathbb{T}} .
$$

For $n \in\left\{n_{0}, n_{0}+1, \ldots\right\}$, by applying mathematical induction, we can obtain the sequence $\left\{u_{n}(t)\right\}_{n \in \mathbb{N}_{1}}$ with

$$
\alpha(t) \leq \alpha_{n}(t) \leq u_{n}(t) \leq u_{n-1}(t) \leq \ldots \leq u_{n_{0}}(t) \leq \beta(t) \text { for } t \in[0, T]_{\mathbb{T}} .
$$

Thus, the condition (iii) holds.

From the above, we obtain that there exists at least one solution of the BVPs (4.3)-(4.4). It still remains to show that $u(t)$ is continuous at $\theta$.

First, it follows from $\lim _{n \rightarrow \infty} u_{n}(0)=\theta$ that there exists an $n_{1} \in\left\{n_{0}, n_{0}+1, \ldots\right\}$ with $\left\|u_{n_{1}}(0)\right\|_{E_{1}}<\frac{\varepsilon}{2}$. Since $u_{n_{1}}(t) \in C\left([0, T]_{\mathbb{T}}, E_{1}\right)$ and $\frac{1}{2^{n}} \in \mathbb{T}$, then we have $\sigma(0)=0$ and there is a $\delta_{n_{1}} \in(0, T)_{\mathbb{T}}$ with $\left\|u_{n_{1}}(t)\right\|_{E_{1}}<\frac{\varepsilon}{2}$ for $t \in\left[0, \delta_{n_{1}}\right)_{\mathbb{T}}$. By the monotonicity of $\left\{u_{n}(t)\right\}_{n \in \mathbb{N}_{0}}$ for each $t \in[0, T]_{\mathbb{T}}$, we have $\|\alpha(t)\|_{E_{1}} \leq\left\|u_{n}(t)\right\|_{E_{1}} \leq$ $\left\|u_{n_{1}}(t)\right\|_{E_{1}}<\frac{\varepsilon}{2}$ for $t \in\left[0, \delta_{n_{1}}\right)_{\mathbb{T}}$ and $n \geq n_{1}$, which means $\|\alpha(t)\|_{E_{1}} \leq\|u(t)\|_{E_{1}}<$ $\frac{\varepsilon}{2}$ for $t \in\left[0, \delta_{n_{1}}\right)_{\mathbb{T}}$. This implies that $u(t)$ is continuous at $\theta$. Consequently, we have completed the proof. 
Note that if we replace $\left[\frac{1}{2^{n+1}}, T-\frac{1}{2^{n+1}}\right]_{\mathbb{T}}$ with $t \in\left[0, T-\frac{1}{2^{n+1}}\right]_{\mathbb{T}}$, then the singularity occurs at $u=\theta$ and $t=T$. If we replace $\left[\frac{1}{2^{n+1}}, T-\frac{1}{2^{n+1}}\right]_{\mathbb{T}}$ with $t \in\left[\frac{1}{2^{n+1}}, T\right]_{\mathbb{T}^{\kappa}}$, then the singularity occurs at $u=\theta$ and $t=0$. If we replace $\left[\frac{1}{2^{n+1}}, T-\frac{1}{2^{n+1}}\right]_{\mathbb{T}}$ with $t \in[0, T]_{\mathbb{T}^{\kappa}}$, then the singularity occurs at $u=\theta$.

REMARK 4.2. Note that if we adjust (A3) appropriately, then one can replace $t \in\left[\frac{1}{2^{n+1}}, T-\frac{1}{2^{n+1}}\right]_{\mathbb{T}}$ in (A1) by

$$
\begin{gathered}
t \in\left[0, T-\frac{1}{2^{n+1}}\right]_{\mathbb{T}}, \\
t \in\left[\frac{1}{2^{n+1}}, T\right]_{\mathbb{T}^{\kappa}},
\end{gathered}
$$

or

$$
t \in[0, T]_{\mathbb{T}^{\kappa}} .
$$

For example, if (4.10) occurs, then (A3) is replaced by

(A3'): There exists a function $\beta(t) \in X$ and $\varphi_{p}\left(\beta^{\Delta}(t)\right) \in C^{\nabla}\left((0, T)_{\mathbb{T}}, E_{1}\right)$ which satisfies $\beta(t) \geq \alpha(t)$ and $\beta(t) \geq \rho_{n_{0}} e$ for $t \in[0, T]_{\mathbb{T}}$ and

$$
\sum_{j=1}^{m_{1}} \phi_{j}\left(\beta\left(\xi_{j}^{*}\right)\right)-\sum_{i=1}^{m_{2}} \psi_{i}\left(\beta^{\Delta}\left(\xi_{i}\right)\right)>0
$$

with

$$
\begin{gathered}
\left(\varphi_{p}\left(\beta^{\Delta}(t)\right)\right)^{\nabla}+q(t) f\left(t, \beta(t), \beta^{\Delta}(t), \ldots, \beta^{\Delta^{n}}(t)\right) \leq \theta \text { for } t \in(0, T)_{\mathbb{T}}, \\
\quad \text { and } \\
\left(\varphi_{p}\left(\beta^{\Delta}(t)\right)\right)^{\nabla}+q(t) f\left(\frac{1}{2^{n_{0}+\mathrm{T}}}, \beta(t), \beta^{\Delta}(t), \ldots, \beta^{\Delta^{n}}(t)\right) \leq \theta \text { for } t \in\left(0, \frac{1}{2^{n_{0}+\mathrm{T}}}\right)_{\mathbb{T}} .
\end{gathered}
$$

Assume that (H1)-(H3), (A1) and (A2) hold. Suppose that the following conditions are satisfied:

$$
\begin{gathered}
\text { (A4): }\left(\varphi_{p}\left(\alpha^{\Delta}(t)\right)\right)^{\nabla}+q(t) f\left(t, u(t), u^{\Delta}(t), \ldots, u^{\Delta^{n}}(t)\right)>\theta \text { for } \\
\left(t, u(t), u^{\Delta}(t), \ldots, u^{\Delta^{n}}(t)\right) \in(0, T)_{\mathbb{T}} \times(0, \alpha(t)] \times E_{1}^{n},
\end{gathered}
$$

where $u(t) \in X$;

(A5): There exists a function $\beta(t) \in X$ and $\varphi_{p}\left(\beta^{\Delta}(t)\right) \in C^{\nabla}\left((0, T)_{\mathbb{T}}, E_{1}\right)$ such that $\beta(t) \geq \rho_{n_{0}} e$ for $t \in[0, T]_{\mathbb{T}}, \quad \sum_{j=1}^{m_{1}} \psi_{i}\left(\beta\left(\xi_{j}^{*}\right)\right)-\sum_{i=1}^{m_{2}} \psi_{i}\left(\beta^{\Delta}\left(\xi_{i}\right)\right)>$ 0 , and

$$
\begin{aligned}
& \left(\varphi_{p}\left(\beta^{\Delta}(t)\right)\right)^{\nabla}+q(t) f\left(t, \beta(t), \beta^{\Delta}(t), \ldots, \beta^{\Delta^{n}}(t)\right) \leq \theta \text { for } t \in(0, T)_{\mathbb{T}}, \\
& \left(\varphi_{p}\left(\beta^{\Delta}(t)\right)\right)^{\nabla}+q(t) f\left(\frac{1}{2^{n_{0}+1}}, \beta(t), \beta^{\Delta}(t), \ldots, \beta^{\Delta^{n}}(t)\right) \leq \theta \text { for } t \in\left(0, \frac{1}{2^{n_{0}+1}}\right)_{\mathbb{T}}, \\
& \quad \text { and } \\
& \quad\left(\varphi_{p}\left(\beta^{\Delta}(t)\right)\right)^{\nabla}+q(t) f\left(T-\frac{1}{2^{n_{0}+1}}, \beta(t), \beta^{\Delta}(t) \ldots, \beta^{\Delta^{n}}(t)\right) \leq \theta \\
& \quad \text { for } t \in\left(T-\frac{1}{2^{n_{0}+1}}, T\right)_{\mathbb{T}} \\
& \text { (A6) } \beta(T) \geq \alpha(T) .
\end{aligned}
$$


Then the conclusion in Theorem 4.1 is also true. This follows immediately from Theorem 4.1 if we show that (A3) holds. Assume that it is not true. In view of (A6), we obtain there is a $\tau_{1} \in(0, T)_{\mathbb{T}}$ such that $\beta(t)-\alpha(t)$ has a local right minimum at $\tau_{1} \in(0, T)_{\mathbb{T}}$, so $(\beta-\alpha)^{\Delta}\left(\tau_{1}\right) \geq \theta$. Using the same reasoning as that of inequality (4.7), we have

$$
\left(\varphi_{p}\left(\alpha^{\Delta}\right)\right)^{\nabla}\left(\tau_{1}\right)-\left(\varphi_{p}\left(\beta^{\Delta}\right)\right)^{\nabla}\left(\tau_{1}\right) \leq \theta
$$

However, by (A4), (A5) and

$$
\alpha\left(\tau_{1}\right)>\beta\left(\tau_{1}\right)>\theta
$$

we get

$$
\left(\varphi_{p}\left(\alpha^{\Delta}\right)\right)^{\nabla}\left(\tau_{1}\right)+q\left(\tau_{1}\right) f\left(\tau_{1}, \beta\left(\tau_{1}\right), \beta^{\Delta}\left(\tau_{1}\right), \ldots, \beta^{\Delta^{n}}\left(\tau_{1}\right)\right)>\theta .
$$

Hence, we deduce

$$
\begin{aligned}
& \left(\varphi_{p}\left(\alpha^{\Delta}\right)\right)^{\nabla}\left(\tau_{1}\right)-\left(\varphi_{p}\left(\beta^{\Delta}\right)\right)^{\nabla}\left(\tau_{1}\right) \\
\geq & \left(\varphi_{p}\left(\alpha^{\Delta}\right)\right)^{\nabla}\left(\tau_{1}\right)+q\left(\tau_{1}\right) f\left(\tau_{1}, \beta\left(\tau_{1}\right), \beta^{\Delta}\left(\tau_{1}\right), \ldots, \beta^{\Delta^{n}}\left(\tau_{1}\right)\right) \\
> & \theta
\end{aligned}
$$

which gives a contradiction with inequality (4.11).

Corollary 4.3. Let $n_{0} \in\{1,2, \ldots\}$ be fixed. Suppose that (H1)-(H3), (A1), (A2) and (A4)-(A6) hold, then the BVPs (1.3)-(1.4) has at least a positive solution $u(t) \in X$ and $\varphi_{p}\left(u^{\Delta}(t)\right) \in C^{\nabla}\left((0, T)_{\mathbb{T}}, E_{1}\right)$ with $u(t) \geq \alpha(t)$, where $t \in[0, T]_{\mathbb{T}}$.

\section{Construction of $\alpha(t)$ and $\beta(t)$}

In this section we will consider how to construct a lower solution $\alpha(t)$ and an upper solution $\beta(t)$ under certain conditions. We assume that

$$
\sum_{i=1}^{m_{2}} \psi_{i}\left(x_{i}\right)-\sum_{j=1}^{m_{1}-1} \phi_{j}\left(x_{j}^{*}\right) \geq 0 \text { for } x_{i}, x_{j}^{*} \in \mathbb{R} .
$$

LEMMA 5.1. If there exists a nonincreasing sequence $\left\{\varepsilon_{n} e\right\} \subset E_{1}$ which satisfies $\varepsilon_{n}>0\left(\varepsilon_{n} \in \mathbb{R}\right)$ and $\lim _{n \rightarrow \infty} \varepsilon_{n}=0$, then there exists a function $\lambda(t) \in$ $C^{\Delta}\left([0, T]_{\mathbb{T}}, E_{1}\right)$ and it satisfies

(i):

$$
\varphi_{p}\left(\lambda^{\Delta}(t)\right) \in C^{\nabla}\left([0, T]_{\mathbb{T}}, E_{1}\right),
$$

$\lambda(t)>\theta$ for $t \in(0, T]_{\mathbb{T}}$ and $\left\|\left(\varphi_{p}\left(\lambda^{\Delta}(t)\right)\right) \nabla\right\|_{E_{1}}>0$ for $t \in(0, T)_{\mathbb{T}} ;$

(ii): $\lambda(0)=\theta, \sum_{j=1}^{m_{1}} \phi_{j}\left(\lambda\left(\xi_{j}^{*}\right)\right)-\sum_{i=1}^{m_{2}} \psi_{i}\left(\lambda^{\Delta}\left(\xi_{i}\right)\right)<0$ and $\theta<\lambda(t) \leq \varepsilon_{n} e$ for $t \in(0, T)_{\mathbb{T}}$.

Proof of Lemma 5.1. Let $L_{n}=\left[\frac{1}{2^{n+1}}, T-\frac{1}{2^{n+1}}\right]_{\mathbb{T}}\left(n \geq n_{0}\right)$. Assume that $r:[0, T]_{\mathbb{T}} \rightarrow\{u \in X \mid u \geq \theta\}$ satisfies

$$
\begin{gathered}
r(0)=\theta, \\
r(t)=\varepsilon_{n}^{p-1} e /(2 T)^{p+1} \text { for } t \in L_{n} \backslash L_{n-1}, n \geq n_{0},
\end{gathered}
$$

and

$$
r(t)=\varepsilon_{n_{0}}^{p-1} e /(2 T)^{p+1} \text { for } t \in\left[\frac{1}{2^{n_{0}}}, T-\frac{1}{2^{n_{0}}}\right]_{\mathbb{T}} .
$$


We define

$$
u(t)=\int_{0}^{t} r(s) \Delta s, v(t)=\left[\int_{0}^{t} u(s) \nabla s\right]^{\frac{1}{p-1}}, w(t)=\int_{0}^{t} v(s) \Delta s .
$$

Assume that $\tau_{2} \in L_{n} \backslash L_{n-1}$ for $n \geq n_{0}$, and $\tau_{3} \in(0, T)_{\mathbb{T}}$ with $\tau_{2}<\tau_{3}$ and $2 \tau_{3}-T \geq \tau_{2}$. It is easy to show that

$$
u, v, w:\left[0, \tau_{2}\right]_{\mathbb{T}} \rightarrow\{u \in X \mid u \geq \theta\}
$$

are continuous and increasing. Denote

$$
a(t)=e\left[c_{0}\left(\tau_{3}-t\right)+c_{1} t\right]^{\frac{1}{p-1}} \text { for } t \in\left[\tau_{2}, T\right]_{\mathbb{T}},
$$

where

$$
c_{0}=-\frac{\tau_{2}}{\tau_{3}} u\left(\tau_{2}\right)+\frac{1}{\tau_{3}}\left(v\left(\tau_{2}\right)\right)^{p-1} \text { and } c_{1}=\frac{\tau_{3}-\tau_{2}}{\tau_{3}} u\left(\tau_{2}\right)+\frac{1}{\tau_{3}}\left(v\left(\tau_{2}\right)\right)^{p-1} .
$$

Hence, we deduce that $a(t)>\theta$ for $t \in\left[\tau_{2}, T\right]_{\mathbb{T}}$ and is nondecreasing. Define

$$
b(t):\left[\tau_{2}, \tau_{3}\right]_{\mathbb{T}} \rightarrow\left\{u \in E_{1} \mid u \geq \theta\right\},
$$

by

$$
\begin{gathered}
b(t)=\int_{\tau_{2}}^{t} a(s) \Delta s+w\left(\tau_{2}\right), \\
B_{1}(t)=\left\{\begin{array}{c}
b(t), t \in\left[\tau_{2}, \tau_{3}\right]_{\mathbb{T}}, \\
b\left(2 \tau_{3}-t\right), t \in\left[\tau_{3}, T\right]_{\mathbb{T}},
\end{array} \quad \lambda(t)=\left\{\begin{array}{c}
w(t), t \in\left[0, \tau_{2}\right]_{\mathbb{T}}, \\
B_{1}(t), t \in\left[\tau_{2}, T\right]_{\mathbb{T}} .
\end{array}\right.\right.
\end{gathered}
$$

It is easy to see that

$$
\begin{gathered}
w\left(\tau_{2}\right)=B_{1}\left(\tau_{2}\right), \quad w^{\Delta}\left(\tau_{2}\right)=B_{1}^{\Delta}\left(\tau_{2}\right), \quad\left(\varphi_{p}\left(w^{\Delta}\right)\right)^{\nabla}\left(\tau_{2}\right)=\left(\varphi_{p}\left(B_{1}^{\Delta}\right)\right)^{\nabla}\left(\tau_{2}\right), \\
w \in C^{\Delta}\left(\left[0, \tau_{2}\right]_{\mathbb{T}}, E_{1}\right), \quad B_{1} \in C^{\Delta}\left(\left[\tau_{2}, T\right]_{\mathbb{T}}, E_{1}\right),
\end{gathered}
$$

and

$$
\varphi_{p}\left(w^{\Delta}\right) \in C^{\nabla}\left(\left[0, \tau_{2}\right]_{\mathbb{T}}, E_{1}\right), \quad \varphi_{p}\left(B_{1}^{\Delta}\right) \in C^{\nabla}\left(\left[\tau_{2}, T\right]_{\mathbb{T}}, E_{1}\right) .
$$

Thus, we know $\lambda \in C^{\Delta}\left([0, T]_{\mathbb{T}}, E_{1}\right)$ and $\varphi_{p}\left(\lambda^{\Delta}\right) \in C^{\nabla}\left([0, T]_{\mathbb{T}}, E_{1}\right)$ with

$$
\left\|\left(\varphi_{p}\left(\lambda^{\Delta}\right)\right)^{\nabla}(t)\right\|_{E_{1}}>0 \text {. }
$$

Since $w(t)>\theta$ for $t \in\left(0, \tau_{2}\right]_{\mathbb{T}}$ and $B_{1}(t)>\theta$ for $t \in\left[\tau_{2}, T\right]_{\mathbb{T}}$, we have $\lambda(t)>\theta$ for $t \in(0, T]_{\mathbb{T}}$. On the other hand, we know that

$$
\begin{aligned}
u\left(\tau_{2}\right) & =\int_{0}^{\tau_{2}} r(s) \Delta s<\frac{\varepsilon_{n}^{p-1} e}{(2 T)^{p}}, \\
v\left(\tau_{2}\right) & =\left[\int_{0}^{\tau_{2}} u(s) \nabla s\right]^{\frac{1}{p-1}}<\frac{\varepsilon_{n} e}{2^{\frac{p}{p-1}} T}, \\
w\left(\tau_{2}\right) & <\tau_{2} \times \frac{\varepsilon_{n} e}{2^{\frac{p}{p-1}} T}<\frac{\varepsilon_{n} e}{2} .
\end{aligned}
$$

By virtue of the monotonicity of $P_{1}(t)$ on $\left[\tau_{2}, \tau_{3}\right]_{\mathbb{T}}$ and $\left[\tau_{3}, T\right]_{\mathbb{T}}$, we have

$$
\begin{aligned}
\lambda\left(\tau_{3}\right) & =\max _{t \in\left[\tau_{2}, T\right]_{\mathbb{T}}} \lambda(t), \\
& =\int_{\tau_{2}}^{\tau_{3}} a(s) \Delta s+w\left(\tau_{2}\right), \\
& \leq\left(\tau_{3}-\tau_{2}\right) \max _{t \in\left[\tau_{2}, \tau_{3}\right]_{\mathbb{T}}} e\left[c_{0}\left(\tau_{3}-t\right)+c_{1} t\right]^{\frac{1}{p-1}}+w\left(\tau_{2}\right),
\end{aligned}
$$




$$
\begin{aligned}
& \leq\left(\tau_{3}-\tau_{2}\right)\left[\left(\tau_{3}-\tau_{2}\right) u\left(\tau_{2}\right)+\left(v\left(\tau_{2}\right)\right)^{p-1}\right]^{\frac{1}{p-1}}+w\left(\tau_{2}\right), \\
& <T\left[T \frac{\varepsilon_{n}^{p-1} e}{(2 T)^{p}}+\frac{\varepsilon_{n}^{p-1} e}{2(2 T)^{p-1}}\right]^{\frac{1}{p-1}}+\frac{\varepsilon_{n} e}{2}<\frac{\varepsilon_{n} e}{2}+\frac{\varepsilon_{n} e}{2}=\varepsilon_{n} e .
\end{aligned}
$$

Hence, we derive

$$
\theta<\lambda(t) \leq \varepsilon_{n} e, \quad t \in(0, T)_{\mathbb{T}} .
$$

From (5.2), we have

$$
0<\phi_{m_{1}-1}(\lambda(t)) \leq \phi_{m_{1}-1}\left(\varepsilon_{n} e\right), t \in(0, T]_{\mathbb{T}} .
$$

Using the inequality

$$
\sum_{i=1}^{m_{2}} \psi_{i}\left(\lambda^{\Delta}\left(\xi_{i}\right)\right)-\sum_{j=1}^{m_{1}-1} \phi_{j}\left(\xi_{j}^{*}\right) \geq \phi_{m_{1}-1}\left(\varepsilon_{n} e\right)>\phi_{m_{1}}\left(\lambda\left(\xi_{m_{1}}^{*}\right)\right)=\phi_{m_{1}}(\lambda(T)),
$$

we obtain

$$
\phi_{m_{1}}\left(\lambda\left(\xi_{m_{1}}^{*}\right)\right)+\sum_{j=1}^{m_{1}-1} \phi_{j}\left(\xi_{j}^{*}\right)-\sum_{i=1}^{m_{2}} \psi_{i}\left(\lambda^{\Delta}\left(\xi_{i}\right)\right)<0 .
$$

Consequently, this completes our proof.

Now we discuss how to construct a lower solution $\alpha(t)$ in (A2) and (A4). Assume that

(A7): for each $n \in\{1,2, \ldots\}$, there exist a constant $k_{0} e \in E$ with $k_{0}>0$ and a strictly monotone decreasing constant sequence $\left\{\rho_{n} e\right\} \subset E$ with $\lim _{n \rightarrow \infty} \rho_{n}=0$, such that

$$
q(t) f\left(t, u(t), u^{\Delta}(t), \ldots, u^{\Delta^{n}}(t)\right) \geq k_{0} e
$$

and

$$
\left(t, u(t), u^{\Delta}(t), \ldots, u^{\Delta^{n}}(t)\right) \in\left[\frac{1}{2^{n+1}}, T-\frac{1}{2^{n+1}}\right]_{\mathbb{T}} \times\left\{u(t) \in X: u(t) \leq \rho_{n} e\right\} \times \mathbb{E}_{1}^{n}
$$

(A8): there exists a function

$$
\beta(t) \in X, \quad \varphi_{p}\left(\beta^{\Delta}(t)\right) \in C^{\nabla}\left((0, T)_{\mathbb{T}}, E_{1}\right),
$$

such that $\beta(t) \geq \theta$ for $t \in[0, T]_{\mathbb{T}}$ and

$$
\begin{gathered}
\sum_{j=1}^{m_{1}} \phi_{j}\left(\beta\left(\xi_{j}^{*}\right)\right)-\sum_{i=1}^{m_{2}} \psi_{i}\left(\beta^{\Delta}\left(\xi_{i}\right)\right)>0, \\
-\left(\varphi_{p}\left(\beta^{\Delta}(t)\right)\right)^{\nabla} \geq q(t) f\left(t, \beta(t), \beta^{\Delta}(t), \ldots, \beta^{\Delta^{n}}(t)\right) \text { for } t \in(0, T)_{\mathbb{T}}, \\
-\left(\varphi_{p}\left(\beta^{\Delta}(t)\right)\right)^{\nabla} \geq q(t) f\left(\frac{1}{2^{n_{0}+1}}, \beta(t), \beta^{\Delta}(t), \ldots, \beta^{\Delta^{n}}(t)\right) \text { for } t \in\left(0, \frac{1}{2^{n_{0}+1}}\right)_{\mathbb{T}}, \\
\quad \text { and } \\
-\left(\varphi_{p}\left(\beta^{\Delta}(t)\right)\right)^{\nabla} \geq q(t) f\left(T-\frac{1}{2^{n_{0}+1}}, \beta(t), \beta^{\Delta}(t), \ldots, \beta^{\Delta^{n}}(t)\right) \text { for } t \in\left(T-\frac{1}{2^{n_{0}+1}}, T\right)_{\mathbb{T}} .
\end{gathered}
$$

TheOREM 5.2. Let $n_{0} \in\{3,4, \ldots\}$ be fixed. If (H1)-(H3), (5.1) and (A7)-(A8) hold, then the BVPs (1.3)-(1.4) has at least a solution $u(t) \in X$ and $\varphi_{p}\left(u^{\Delta}(t)\right) \in$ $C^{\nabla}\left((0, T)_{\mathbb{T}}, E_{1}\right)$ with $u(t) \geq \alpha(t)$, where $t \in[0, T]_{\mathbb{T}}$. 
Proof of Theorem 5.2. By Corollary 4.3, we only need to show that conditions (A1), (A2), and (A4)-(A6) are satisfied. Without loss of generality, we suppose that

$$
\beta(t)>\rho_{n_{0}} e \text { for } t \in[0, T]_{\mathbb{T}} \text { and } \sum_{j=1}^{m_{1}} \phi_{j}\left(\beta\left(\xi_{j}^{*}\right)\right)-\sum_{i=1}^{m_{2}} \psi_{i}\left(\beta^{\Delta}\left(\xi_{i}\right)\right)>\rho_{n_{0}} .
$$

By virtue of (A7), (A8) and (5.3), we know that (A1) and (A5) hold.

From Lemma 5.1 there exists a function $\lambda(t) \in C^{\Delta}\left([0, T]_{\mathbb{T}}, X\right)$ satisfying

(i): $\varphi_{p}\left(\lambda^{\Delta}(t)\right) \in C^{\nabla}\left([0, T]_{\mathbb{T}}, E_{1}\right), \lambda(t)>\theta$ for $t \in(0, T]_{\mathbb{T}}$ and

$$
R_{1}=\left\|\left(\varphi_{p}\left(\lambda^{\Delta}(t)\right)\right)^{\nabla}\right\|_{E_{1}}>0
$$

(ii): $\lambda(0)=\theta, \sum_{j=1}^{m_{1}} \phi_{j}\left(\lambda\left(\xi_{j}^{*}\right)\right)-\sum_{i=1}^{m_{2}} \psi_{i}\left(\lambda^{\Delta}\left(\xi_{i}\right)\right)<0$ and $\lambda(t) \leq \rho_{n} e$ for $t \in(0, T)_{\mathbb{T}}$.

Assume that

$$
m=\min \left\{e,\left(\frac{k_{0} e}{2 R_{1}}\right)^{1 /(p-1)}, \frac{\rho_{n_{0}} e}{\|\lambda(t)\|_{E_{1}}}\right\} .
$$

Let $\alpha(t)=m \lambda(t)$ for $t \in[0, T]_{\mathbb{T}}$. Then we have $\alpha(t) \in X, \varphi_{p}\left(\alpha^{\Delta}(t)\right) \in C^{\nabla}\left((0, T)_{\mathbb{T}}, E_{1}\right)$ and $\alpha(0)=\theta$ with $\theta<\alpha(t) \leq \lambda(t) \leq \rho_{n} e$ for $t \in\left(\frac{1}{2^{n+1}}, T\right]_{\mathbb{T}}$. Furthermore, we have

$$
\sum_{j=1}^{m_{1}} \phi_{j}\left(\alpha\left(\xi_{j}^{*}\right)\right)-\sum_{i=1}^{m_{2}} \psi_{i}\left(\alpha^{\Delta}\left(\xi_{i}\right)\right)<0 .
$$

For an arbitrary

$$
\left(t, u, u^{\Delta}, \ldots, u^{\Delta^{n}}\right) \in(0, T]_{\mathbb{T}} \times(0, \alpha(t)] \times E_{1}^{n},
$$

there exists an $n \in\left\{n_{0}, n_{0}+1, \ldots\right\}$ such that

$$
\left(t, u, u^{\Delta}, \ldots u^{\Delta^{n}}\right) \in\left[\frac{1}{2^{n+1}}, T-\frac{1}{2^{n+1}}\right]_{\mathbb{T}} \times(0, \alpha(t)] \times E_{1}^{n} .
$$

So we have

$$
\begin{aligned}
& q(t) f\left(t, u(t), u^{\Delta}(t), \ldots, u^{\Delta^{n}}(t)\right)+\left(\varphi_{p}\left(\alpha^{\Delta}(t)\right)\right)^{\nabla} \\
\geq & k_{0} e+\left(\varphi_{p}\left(m \lambda^{\Delta}(t)\right)\right)^{\nabla}, \\
= & k_{0} e+m^{p-1}\left(\varphi_{p}\left(\lambda^{\Delta}(t)\right)\right)^{\nabla}, \\
\geq & k_{0} e-m^{p-1}\left\|\left(\varphi_{p}\left(\lambda^{\Delta}(t)\right)\right)^{\nabla}\right\|_{E_{1}}, \\
\geq & k_{0} e-\frac{k_{0} e}{2 R_{1}}\left\|\left(\varphi_{p}\left(\lambda^{\Delta}(t)\right)\right)^{\nabla}\right\|_{E_{1}}, \\
= & \frac{k_{0} e}{2}>\theta .
\end{aligned}
$$

Thus, we see that (A4) holds and (A2) is true if $u(t)=\alpha(t)$. Since $\alpha(T) \leq$ $\|\alpha(t)\|_{E_{1}}=m\|\lambda(t)\|_{E_{1}} \leq \rho_{n_{0}}$, we have $\beta(T) \geq \rho_{n_{0}} \geq \alpha(T)$, so (A6) is fulfilled. By Corollary 4.3, the BVPs (1.3)-(1.4) has at least a solution

$$
u(t) \in X, \quad \varphi_{p}\left(u^{\Delta}(t)\right) \in C^{\nabla}\left((0, T)_{\mathbb{T}}, E_{1}\right) \text { with } u(t)>\theta,
$$

where $t \in[0, T]_{\mathbb{T}}$.

According to Theorem 5.2, it may be difficulty for us to construct $\beta$ in a straightforward way due to the assumption (A8). The following theorem may enable us to find $\beta$ by replacing (A8) with a simply verified condition. 
TheOREM 5.3. Let $n_{0} \in\{1,2, \ldots\}$ be fixed. Suppose that (H1)-(H3), (A1) and (A2) hold, and that the following conditions are satisfied:

$M_{1} e>\theta$ and $M_{2} e>\max \left\{\alpha(t), \rho_{n_{0}} e\right\}$, where $M_{1}, M_{2} \in \mathbb{R}$,

$$
\left\{\begin{array}{l}
q(t) f\left(t, M_{1} e t+M_{2} e, M_{1} e, \theta, \ldots, \theta\right) \leq \theta \text { for } t \in(0, T)_{\mathbb{T}} \\
q(t) f\left(\frac{1}{2^{n_{0}+1}}, M_{1} e t+M_{2} e, M_{1} e, \theta, \ldots, \theta\right) \leq \theta \text { for } t \in\left(0, \frac{1}{2^{n_{0}+1}}\right)_{\mathbb{T}} \\
q(t) f\left(\frac{1}{2^{n_{0}+1}}, M_{1} e t+M_{2} e, M_{1} e, \theta, \ldots, \theta\right) \leq \theta \text { for } t \in\left(T-\frac{1}{2^{n_{0}+1}}, T\right)_{\mathbb{T}}
\end{array}\right.
$$

and

$$
\sum_{j=1}^{m_{1}} \phi_{j}\left(M_{1} e \xi_{j}^{*}+M_{2} e\right)-\sum_{i=1}^{m_{2}} \psi_{i}\left(M_{1} e\right)>0 .
$$

Then the BVPs (1.3)-(1.4) has at least a solution

$$
u(t) \in X, \varphi_{p}\left(u^{\Delta}(t)\right) \in C^{\nabla}\left((0, T)_{\mathbb{T}}, E_{1}\right) \text { with } u(t)>\theta,
$$

where $t \in[0, T]_{\mathbb{T}}$.

Proof of Theorem 5.3. Denote $\beta(t)=M_{1}$ et $+M_{2} e$ for $t \in[0, T]_{\mathbb{T}}$, then we have $\beta(t) \in X, \varphi_{p}\left(\beta^{\Delta}(t)\right) \in C^{\nabla}\left((0, T)_{\mathbb{T}}, E_{1}\right)$ and $\beta(t) \geq \alpha(t)$ and $\beta(t) \geq \rho_{n_{0}} e$ for $t \in[0, T]_{\mathbb{T}}$. A direct calculation gives

$$
\begin{aligned}
& \qquad \sum_{j=1}^{m_{1}} \psi_{i}\left(\beta\left(\xi_{j}^{*}\right)\right)-\sum_{i=1}^{m_{2}} \psi_{i}\left(\beta^{\Delta}\left(\xi_{i}\right)\right)>0, \\
& \qquad\left(\varphi_{p}\left(\beta^{\Delta}(t)\right)\right)^{\nabla}+q(t) f\left(t, \beta(t), \beta^{\Delta}(t), \ldots \beta^{\Delta^{n}}(t)\right) \leq \theta \text { for } t \in(0, T)_{\mathbb{T}}, \\
& \left(\varphi_{p}\left(\beta^{\Delta}(t)\right)\right)^{\nabla}+q(t) f\left(\frac{1}{2^{n_{0}+1}}, \beta(t), \beta^{\Delta}(t), \ldots \beta^{\Delta^{n}}(t)\right) \leq \theta \text { for } t \in\left(T-\frac{1}{2^{n_{0}+1}}, \frac{1}{2^{n_{0}+1}}\right)_{\mathbb{T}}, \\
& \text { and } \\
& \qquad\left(\varphi_{p}\left(\beta^{\Delta}(t)\right)\right)^{\nabla}+q(t) f\left(\frac{1}{2^{n_{0}+1}}, \beta(t), \beta^{\Delta}(t), \ldots \beta^{\Delta^{n}}(t)\right) \leq \theta \text { for } t \in\left(0, \frac{1}{2^{n_{0}+1}}\right)_{\mathbb{T}} .
\end{aligned}
$$

This implies that (A3) holds. By virtue of Theorem 4.1, we complete the proof.

From Theorems 5.2 and 5.3 we can obtain the following theorem directly.

TheOrem 5.4. Let $n_{0} \in\{1,2, \ldots\}$ be fixed. Suppose that $(\mathrm{H} 1)-(\mathrm{H} 3),(5.1)$ and (A7) hold, and that there exist real constants $M_{1} e, M_{2} e \in E_{1}$ such that (5.4) and (5.5) are true, where $M_{1}$ and $M_{2}$ are positive. Then the BVPs (1.3)-(1.4) has at least a solution $u(t) \in X, \varphi_{p}\left(u^{\Delta}(t)\right) \in C^{\nabla}\left((0, T)_{\mathbb{T}}, E_{1}\right)$ and $u(t)>\theta$ for $t \in(0, T]_{\mathbb{T}}$.

Proof OF TheOrem 5.4. Without loss of generality, we suppose that $\rho_{n_{0}} e<$ $M_{2} e$. From (A7), we know that (A1) holds and

$$
M_{2} e>\rho_{n_{0}} e>\rho_{n_{0}+1} e>\ldots \text { and } \lim _{n \rightarrow \infty} \rho_{n} e=\theta .
$$

By using a similar argument as that of Theorem 5.2, there exists a function $\alpha(t) \in$ $X$, and $\varphi_{p}\left(\alpha^{\Delta}(t)\right) \in C^{\nabla}\left((0, T)_{\mathbb{T}}, E_{1}\right)$ with $\alpha(0)=\theta$,

$$
\sum_{j=1}^{m_{1}} \phi_{j}\left(\alpha\left(\xi_{j}^{*}\right)\right)-\sum_{i=1}^{m_{2}} \psi_{i}\left(\alpha^{\Delta}\left(\xi_{i}\right)\right)<0
$$

and $\alpha(t)>\theta$ for $t \in(0, T]_{\mathbb{T}}$, such that

$$
\left(\varphi_{p}\left(\alpha^{\Delta}(t)\right)\right)^{\nabla}+q(t) f\left(t, \alpha(t), \alpha^{\Delta}(t), \ldots, \alpha^{\Delta^{n}}(t)\right) \geq \theta
$$

for $t \in(0, T)_{\mathbb{T}}$ and $\alpha(t) \leq \rho_{n_{0}} e$. This together with (5.6) gives that

$$
M_{2}>\max \left\{\alpha(t), \rho_{n_{0}} e\right\} .
$$


This implies that all conditions of Theorem 5.3 are fulfilled. Consequently, we complete the proof.

\section{One Example}

In this section, we present an example to illustrate our main results.

Let $E_{1}=l^{\infty}=\left\{u=\left(u_{1}, u_{2}, \ldots, u_{n}, \ldots\right)\left|\sup _{n}\right| u_{n} \mid<+\infty\right\}$ with norm $\|u\|_{E_{1}}=$ $\sup _{n}\left|u_{n}\right|$ and $E_{1}$ is a finite dimensional space. Define

$$
P_{1}=\left\{u=\left(u_{1}, u_{2}, \ldots, u_{n}, \ldots\right) \mid u_{n} \geq 0, n=1,2, \ldots\right\},
$$

then $P_{1}$ is a normal cone in the compact Banach space $E_{1}$.

Assume that

$$
\mathbb{T}=\{0\} \cup\left\{(1 / 2)^{\mathbb{N}}\right\} \cup[1 / 2,1] .
$$

Consider the following boundary value problem in $E_{1}$ of the form:

$$
\begin{aligned}
& \left(\varphi_{4}\left(u^{\Delta}(t)\right)\right)^{\nabla}+q(t) f\left(t, u(t), u^{\Delta}(t)\right)=\theta \text { for } t \in(0,1)_{\mathbb{T}}, \\
& u(0)=\theta, u(1)+\frac{1}{5} u\left(\frac{1}{8}\right)-\frac{1}{5} u^{\Delta}\left(\frac{1}{8}\right)-\frac{1}{10} u^{\Delta}\left(\frac{1}{4}\right)-\frac{1}{5} u^{\Delta}\left(\frac{3}{4}\right)-\frac{1}{10} u^{\Delta}(1)=0 .
\end{aligned}
$$

It is obvious that $T=1, p=4, \varphi_{1}(x)=\psi_{1}(x)=\frac{1}{5} x, \psi_{2}(x)=\frac{1}{10} x, \psi_{3}(x)=\frac{1}{5} x$ and $\psi_{4}(x)=x$. Denote $q(t)=t^{4}+4$ and

$$
f\left(t, u, u^{\Delta}\right)=\frac{t e}{u^{7}(t)}+u^{8}(t)+\left(t u^{\Delta}(t)\right)^{2}-\lambda^{2} e, t \in[0,1]_{\mathbb{T}}, u \in E_{1}, \lambda \in \mathbb{R},
$$

where $\lambda^{2} \geq 10$ and $e=(1,1, \ldots, 1 \ldots)$. Letting

$$
n_{0} \in\{1,2, \ldots\}, \rho_{n}=\left(\frac{1}{2^{n+1}\left(\lambda^{2}+a_{1}\right)}\right)^{1 / 7}
$$

and $k_{0}=a_{1}>0$, we have $\rho_{n_{0}} \leq 1$. Note that $\left(H_{1}\right)-\left(H_{3}\right)$ and $(5.1)$ hold. For $n \in\{1,2, \ldots\}, t \in\left[\frac{1}{2^{n+1}}, 1-\frac{1}{2^{n+1}}\right]_{\mathbb{T}}$ and $\theta<u \leq \rho_{n} e$, we have

$$
\begin{aligned}
& q(t) f\left(t, u, u^{\Delta}\right) \\
\geq & \left(t^{4}+4\right)\left(\frac{e}{2^{n+1} \rho_{n}^{7}}-\lambda^{2} e\right), \\
\geq & \left(t^{4}+4\right)\left(\lambda^{2} e+a_{1} e-\lambda^{2} e\right), \\
> & a_{1} e>\theta,
\end{aligned}
$$

which indicates that (A7) is satisfied.

Now we show that $(\mathrm{A} 8)$ holds with $\beta(t)=t^{1 / 7} e, t \in[0,1]_{\mathbb{T}}$. Notice that if $t \in(1 / 2,1]_{\mathbb{T}}$, then we have

and

$$
\begin{aligned}
& \beta^{\Delta}(t)=\beta^{\prime}(t)=\frac{1}{7} t^{-6 / 7} e, \\
& \varphi_{4}\left(\beta^{\Delta}(t)\right)=\left(\frac{1}{343} t^{-\frac{18}{7}}\right) e,
\end{aligned}
$$

$$
\left(\varphi_{4}\left(\beta^{\Delta}(t)\right)\right)^{\nabla}=-\left(\frac{18}{2401} t_{1}^{-\frac{25}{7}}\right) e \leq \theta .
$$

If $t=1 / 2$, then we get

$$
\begin{aligned}
\beta^{\Delta}(t) & =\frac{1}{7} t^{-6 / 7} e, \\
\varphi_{4}\left(\beta^{\Delta}(t)\right) & =\left(\frac{1}{343} t^{-\frac{18}{7}}\right) e,
\end{aligned}
$$


and

$$
\left(\varphi_{4}\left(\beta^{\Delta}(t)\right)\right)^{\nabla}=\left(\frac{2}{343}\left(\frac{1}{2}\right)^{-\frac{18}{7}}-\frac{2}{343}\right) e \approx-0.1714 e \leq \theta .
$$

When $t=1 / 2^{n}(n=2,3, \ldots)$, we deduce that $\sigma(t)=2 t, \rho(t)=\frac{t}{2}, \mu(t)=t$, $\nu(t)=\frac{t}{2}$, and

$$
\beta^{\Delta}(t)=\frac{1}{t}\left[(2 t)^{1 / 7}-t^{1 / 7}\right] e, \varphi_{4}\left(\beta^{\Delta}(t)\right)=\frac{1}{t^{3}}\left[(2 t)^{1 / 7}-t^{1 / 7}\right]^{3} e .
$$

By induction, we can derive that

$$
\begin{aligned}
& \left(\varphi_{4}\left(\beta^{\Delta}(t)\right)\right)^{\nabla} \\
= & 2^{4 n+1}\left[\left(\frac{1}{2^{n-1}}\right)^{1 / 7}-\left(\frac{1}{2^{n}}\right)^{1 / 7}\right]^{3} e-2^{4 n+4}\left[\left(\frac{1}{2^{n}}\right)^{1 / 7}-\left(\frac{1}{2^{n+1}}\right)^{1 / 7}\right]^{3} e, \\
\leq & \theta .
\end{aligned}
$$

Thus, for $t \in(0,1]_{\mathbb{T}}$ we have

$$
\begin{aligned}
& \left(\varphi_{4}\left(\beta^{\Delta}(t)\right)\right)^{\nabla}+q(t) f\left(t, \beta(t), \beta^{\Delta}(t)\right) \\
& \leq \begin{cases}\left(t^{4}+4\right)\left(\frac{t}{t} e+t^{\frac{8}{7}} e+\left(\frac{1}{7} t^{1 / 7}\right)^{2} e-\lambda^{2} e\right), & t \in\left[\frac{1}{2}, 1\right]_{\mathbb{T}}, \\
\left(t^{4}+4\right)\left(\frac{t}{t} e+t^{\frac{8}{7}} e+\left((2 t)^{1 / 7}-t^{1 / 7}\right)^{2} e-\lambda^{2} e\right), & t \in\left\{1 / 2^{n}, n=2,3, \ldots,\right\},\end{cases} \\
& \leq\left(t^{4}+4\right)\left(10 e-\lambda^{2} e\right),
\end{aligned}
$$

For $t \in\left(0, \frac{1}{2^{n_{0}+\mathrm{T}}}\right)_{\mathbb{T}}$, we have

$$
\begin{aligned}
& \left(\varphi_{4}\left(\beta^{\Delta}(t)\right)\right)^{\nabla}+q(t) f\left(\frac{1}{2^{n_{0}+1}}, \beta(t), \beta^{\Delta}(t)\right) \\
& \leq\left(t^{4}+4\right)\left(\frac{1}{2^{n_{0}+1} t} e+\left(t^{1 / 7}\right)^{8} e+\left((2 t)^{1 / 7}-t^{1 / 7}\right)^{2} e-\lambda^{2} e\right), \\
& \leq\left(t^{4}+4\right)\left(10 e-\lambda^{2} e\right), \\
& \leq \theta .
\end{aligned}
$$

Moreover, we obtain

$$
\beta(1)+\varphi_{1}\left(\beta\left(\frac{1}{8}\right)\right)-\psi_{1}\left(\beta^{\Delta}\left(\frac{1}{8}\right)\right)-\psi_{2}\left(\beta\left(\frac{1}{4}\right)\right)-\psi_{3}\left(\beta^{\Delta}\left(\frac{3}{4}\right)\right)-\psi_{4}(\beta(1))>0 .
$$

Hence, all conditions of Theorem 5.2 are satisfied. As a result, the problem (6.1) has at least a solution $u \in E_{1}=l^{\infty}$ with $\varphi_{4}\left(u^{\Delta}(t)\right) \in C^{\nabla}\left((0, T)_{\mathbb{T}}, E_{1}\right)$ and $u(t)>\theta$ for $t \in(0, T]_{\mathbb{T}}$.

\section{References}

[1] S. Hilger, Ein Maßkettenkalkül mit Anwendung auf Zentrumsmannigfaltigkeiten (in German), Universität Würzburg, 1988.

[2] B. Jain and A. D. Sheng, An exploration of the approximation of derivative functions via finite differences, Rose-Hulman Undergraduate Math J. 8 (2007), 1-19.

[3] F.M. Atici, D.C. Biles and A. Lebedinsky, An application of time scales to economics, Math. Comput. Modelling, 43 (2006), 718-726.

[4] Q. Sheng, An exploration of combined dynamic derivatives on time scales for computational applications, Nonlinear Anal. (RWA), 7 (2006), 396-414.

[5] D.M. Thomas, L. Vandemuelebroeke and K. Yamaguchi, A mathematical evolution model for phytoremediation of metals, Discrete Contin. Dyn. Syst. Ser. B 5 (2005), 411-422.

[6] N.A. Hamal and F. Yoruk, Positive solutions of nonlinear $m$-point boundary value problems on time scales, J. Computat. Appl. Math. 231 (2009), 92-105. 
[7] S. Li, Y.H. Su and Z. Feng, Positive solutions to $p$-Laplacian multi-point BVPs on time scales, Dyn. Partial Differ. Equ. 7 (2010), 45-64.

[8] Y.H. Su, Multiple positive pseudo-symmetric solutions of $p$-Laplacian dynamic equations on time scales, Math. Comput. Modelling, 49 (2009), 1664-1681.

[9] Y.H. Su, W.T. Li and H.R. Sun, Positive solutions of singular $p$-Laplacian BVPs with sign changing nonlinearity on time scales, Math. Comput. Modelling, 48 (2008), 845-858.

[10] Y.H. Su, B. Li and C.Y. Huang, Positive solution to a singular $p$-Laplacian BVP with signchanging nonlinearity involving derivative on time scales, Adv. Difference Equ. 2009 Article ID 623932, 21 pages.

[11] Y.H. Su and W.T. Li, Triple positive symmetric solutions of two point BVPs for $p$-Laplacian dynamic equations on time scales, Acta. Math. Scientia (in Chinese), 28 (2008), 51-60.

[12] Y.H. Su and W.T. Li, Triple positive solutions of $m$-point BVPs for $p$-Laplacian dynamic equations on time scales, Nonlinear Anal. 69 (2008), 3811-3820.

[13] H.R. Sun and W.T. Li, Existence theory for positive solutions to one-dimensional $p$-Laplacian boundary value problems on time scales, J. Diff. Equ. 240 (2007), 217-248.

[14] H. Lü, D. O'Regan and R.P. Agarwal, Upper and lower solutions for the singular $p$-Laplacian with sign changing nonlinearities and nonlinear boundary data, J. Comput. Appl. Math. 181 (2005), 442-466.

[15] H. Lü, D. O'Regan and R.P. Agarwal, On the existence of multiple periodic solutions for the vector $p$-Laplacian via critical point theory, Appl. Math. 50 (2005), 555-568.

[16] D. O'Regan, Upper and lower solutions for the singular problems arising in the theory of membrane response of a sphercial cap, Nonlnear Anal. 47 (2001), 1163-1174.

[17] M. Cichon, I.Kubiaczyk, A. Sikorska-Nowak and A. Yantir, Weak solutions for the dynamic Cauchy problem in Banach spaces, Nonlinear Anal. 71 (2009), 2936-2943.

[18] J. G. Dix and G.L. Karakostas, A fixed-point theorem for $S$-type operators on Banach spaces and its applications to boundary-value problems, Nonlinear Anal. 71 (2009), 3872-3880.

[19] X. Zhang, M. Feng and W. Ge, Existence of solutions of boundary value problems with integral boundary conditions for second-order impulsive integro-differential equations in Banach spaces, J. Comput. Appl. Math. 233 (2010), 1915-1926.

[20] D. Guo, Y.J. Cho and J. Zhu, Partial Ordering Methods In Nonlinear Problems, Nova Science Publishers, New York, 2004.

[21] E. Zeidler, Nonlinear Functional Analysis and its Applications I: Fixed-Point Theorems, Springer-Verlag, New York, 1993.

[22] K. Deimling, Nonlinear Functional Analysis, Spring-Verlag, Berlin Heidelberg, 1985.

[23] W.B. Liu and Z. Feng, Periodic solutions for $p$-Laplacian systems of Liénard-type, Commun. Pure Appl. Anal. 10 (2011), 1410-1418.

[24] M. Bohner and A. Peterson, Advances in Dynamic Equation on Time Scales, Birkhäuser, Boston, 2003.

[25] M. Bohner and A. Peterson, Dynamic Equation on Time Scales, An Introduction with Applications, Birkhäuser, Boston, 2001.

[26] B.P. Rynne, $L^{2}$ spaces and boundary value problems on time-scales, J. Math. Anal. Appl. 328 (2007), 1217-1236.

School of Mathematics and Physics, Xuzhou University of Technology, Xuzhou, JiANGSU 221008, CHINA

Department of Mathematics, University of Texas-Pan American, Edinburg, TX 78539, USA

E-mail address: zsfeng@utpa.edu 